\title{
Gravitomagnetic tidal currents in rotating neutron stars
}

\author{
Eric Poisson and Jean Douçot \\ Department of Physics, University of Guelph, Guelph, Ontario, N1G 2W1, Canada
}

(Dated: January 25, 2017)

\begin{abstract}
It was recently revealed that a rotating compact body responds dynamically when it is subjected to a gravitomagnetic tidal field, even when this field is idealized as time-independent. The dynamical response is characterized by time-changing internal currents, and it was suspected to originate from zero-frequency $g$-modes and $r$-modes driven by the tidal forces. In this paper we provide additional insights into the phenomenon by examining the tidal response of a rotating body within the framework of post-Newtonian gravity. This approach allows us to develop an intuitive picture for the phenomenon, which relies on the close analogy between post-Newtonian gravity and Maxwell's theory of electromagnetism. In this picture, the coupling between the gravitomagnetic tidal field and the body's rotational velocity is naturally expected to produce an unbalanced Lorentz-like force within the body, and it is this force that is responsible for the tidal currents. The simplicity of the fluid equations in the post-Newtonian setting allows us to provide a complete description of the zero-frequency modes and demonstrate their precise role in the establishment of the tidal currents. We estimate the amplitude of these currents, and find that for neutron-star binaries of relevance to LIGO, the scale of the velocity perturbation is measured in kilometers per second when the rotation period is comparable to 100 milliseconds. This estimate indicates that the tidal currents may have a significant impact on the physics of neutron stars near merger.
\end{abstract}

\section{INTRODUCTION}

The tidal interaction between neutron stars in a close binary system has recently been the subject of intense investigation, following the remarkable observation [1, 2] that the tidal deformation of each body could have a measurable impact on the emitted gravitational waves. The effect depends on the tidal polarizability of each neutron star, and a large effort was devoted to the computation of relativistic Love numbers 3 -5 for realistic models of neutron stars, and to ascertain the importance of the tidal deformation on the gravitational-wave signal [6] 26]. While this work was restricted to the regime of static tides (or adiabatic tides), in which the external, orbital time scale is long compared with the internal, hydrodynamical time scale of the neutron star, an extension to the regime of dynamical tides was recently developed [27 30], following the pioneering work of Flanagan and Hinderer [1]. The dynamical aspects of the tidal interaction, during which the body's internal fluid modes are driven by the external tidal forces, were shown to be significant for binaries with mass ratios up to approximately 3 , when they implicate stiff neutron stars with large radii [30].

An unexpected aspect of the tidal dynamics of compact binaries was recently revealed by Landry and Poisson [31. These authors demonstrated that a rotating compact body responds dynamically when it is subjected to a gravitomagnetic tidal field - the inhomogeneous piece of the gravitational field produced by the mass current associated with the orbital motion of the companion body. Most strikingly, the phenomenon was revealed in the idealization in which the gravitomagnetic tidal field is taken to be stationary; in this case the tidal interaction produces an internal velocity field that grows linearly with time. The phenomenon was attributed to zero-frequency fluid modes, which do not provide a restoring force that would balance out the external tidal forces and keep the fluid stationary.

The analysis presented in Ref. 31 was performed within a perturbative context in which the tidal forces are weak and idealized as time-independent, and in which the body is only allowed to rotate slowly. The calculations, however, were carried out in full general relativity, and therefore incorporated all strong-field effects in the interior of the compact body. The intrinsic complexity of the computations prevented these authors from developing an intuitive physical picture for the phenomenon, and kept them from assessing its significance. In particular, the zero-frequency modes were presented as a likely culprit for the phenomenon, but their precise role could not be ascertained.

In this paper we provide the physics insights that were missing from the original analysis. Our strategy is to approach the problem anew in the framework of post-Newtonian gravity, assuming that the internal gravity of the compact body is not too strong. While the predictions of this analysis are likely to have limited accuracy from a quantitative point of view, they will be qualitative robust, and they come with an intuitive understanding that was not easily accessible in the general-relativistic treatment. We retain the assumption that the tidal forces are weak, but we no longer rely on the stationary idealization; our tidal field can now vary with time, on a time scale that's assumed to be long compared with the body's internal, hydrodynamical time scale. We also retain the assumption that the body is rotating slowly.

In this post-Newtonian setting, the generation of gravitomagnetic tidal currents inside a rotating neutron star can 
be revealed with a relatively simple analysis. More importantly, the close analogy between post-Newtonian gravity and Maxwell's electromagnetism provides us with a strong intuitive basis. The phenomenon no longer looks so mysterious when viewed in this particular way.

We may now state the problem more precisely, and develop the intuition behind the phenomenon. We consider a body of mass $M$, radius $R$, and angular velocity $\Omega$ immersed in a gravitomagnetic tidal environment created by a remote companion of mass $M^{\prime}$ moving with velocity $v^{\prime}$ on an orbit of radius $r^{\prime}$. Just as an orbiting electric charge would create a magnetic field, the orbiting companion creates a gravitomagnetic field $\boldsymbol{B}$ around the body. The inhomogeneous piece of this field, the one responsible for the tidal interaction, scales as $G M v^{\prime} \boldsymbol{x} / r^{\prime 3}$, in which $\boldsymbol{x}$ is the position from the body's center-of-mass. The tidal field couples to the body's rotational velocity $\boldsymbol{v}=\boldsymbol{\Omega} \times \boldsymbol{x}$ and creates, inside the body, a force density given by

$$
\boldsymbol{f}=\frac{1}{c^{2}} \rho \boldsymbol{v} \times \boldsymbol{B}
$$

the gravitational analogue of the Lorentz force. Unlike the typical situation encountered in Newtonian tides, or in general relativistic, gravitoelectric tides, this force is not balanced out by pressure-gradient forces within the fluid. Instead, the gravitomagnetic tidal forces act on the body and establish a velocity perturbation $\delta \boldsymbol{v}$ proportional to the time integral of the tidal field. In the idealization of a time-independent $\boldsymbol{B}$, the velocity field would grow linearly in time, just as revealed in Ref. [31. In the more realistic case of a time-changing $\boldsymbol{B}, \delta \boldsymbol{v}$ is modulated by the changes in the tidal environment.

The root of the phenomenon is therefore an unbalanced force that arises from the coupling between the gravitomagnetic tidal field and the body's rotational velocity. These elementary considerations imply that the velocity perturbation must scale as

$$
\delta v=\frac{G M^{\prime} v^{\prime} \Omega R^{2}}{c^{2} \omega^{\prime} r^{\prime 3}}
$$

where $\omega^{\prime}:=v^{\prime} / r^{\prime}$ is the orbital angular velocity, which corresponds to the frequency of oscillation of the tidal field. In this expression, the factor $G M^{\prime} / r^{\prime 3}$ indicates that the effect is the result of a tidal interaction, the factor $v^{\prime} / c^{2}$ further reveals that it is a post-Newtonian, gravitomagnetic effect, the factor $\Omega R$ shows that the effect results from the coupling with the body's rotational velocity, the factor $R$ comes from the scaling of $\boldsymbol{B}$ with the position relative to the body's center-of-mass, and the last factor $1 / \omega^{\prime}$ comes from the time integral of the force density.

The scale of the velocity perturbation can be re-expressed in terms of the masses $M$ and $M^{\prime}$, the body's rotation period $P:=2 \pi / \Omega$ and radius $R$, and the orbital frequency $f^{\prime}:=\omega^{\prime} /(2 \pi)$. We rely on Kepler's law $\omega^{\prime 2}=G\left(M+M^{\prime}\right) / r^{\prime 3}$ to eliminate the orbital radius $r^{\prime}$, and get

$$
\delta v=\frac{(2 \pi)^{7 / 3} G^{1 / 3}}{c^{2}} \frac{M^{\prime}}{\left(M+M^{\prime}\right)^{2 / 3}} \frac{R^{2}}{P} f^{4 / 3} .
$$

Inserting fiducial values for a typical binary system of neutron stars near merger, we find that the scale of the velocity perturbation is given by

$$
\delta v=2\left(\frac{M^{\prime}}{1.4 M_{\odot}}\right)\left(\frac{2.8 M_{\odot}}{M+M^{\prime}}\right)^{2 / 3}\left(\frac{R}{12 \mathrm{~km}}\right)^{2}\left(\frac{100 \mathrm{~ms}}{P}\right)\left(\frac{f}{100 \mathrm{~Hz}}\right)^{4 / 3} \mathrm{~km} / \mathrm{s} .
$$

The amplitude of the tidal currents is measured in kilometers per second, and should therefore be significant in these systems.

In the remaining sections of the paper we give a precise statement of these results and provide a complete derivation, taking the compact body to be a rigidly rotating perfect fluid, and considering generic tidal environments. As we stated previously, our treatment is based on four key assumptions. First, we take the gravitational field inside the body to be sufficiently weak to permit a post-Newtonian approximation carried out to the first order. Second, we assume that the tidal perturbation is small and can be adequately described by a first-order perturbative treatment. Third, we assume that the gravitomagnetic tidal field changes on a time scale that is long compared with the body's internal, hydrodynamical time scale. And fourth, we assume that the body rotates slowly, so that all equations can be linearized with respect to the angular velocity $\Omega$.

We begin in Sec. II with a presentation of those aspects of post-Newtonian gravity that are relevant for our purposes. In particular, we introduce the vector potential $\boldsymbol{U}$ associated with the gravitomagnetic field, and describe a generic gravitomagnetic tidal environment in terms of a symmetric-tracefree tensor $\mathcal{B}_{a b}(t)$. In Sec. III we introduce the post-Newtonian version of Euler's equation, which governs the behavior of a perfect fluid. We first integrate this equation for the unperturbed configuration of a nonrotating star, and then switch on the tidal field and the rotation. 
We find that the perturbation equations take the same form as those governing a nonrotating fluid in Newtonian gravity, but with a driving force that couples the gravitomagnetic tidal field to the body's rotational velocity. In Sec. IV we recast the perturbation equations in a convenient form involving a Lagrangian displacement vector $\boldsymbol{\xi}$. This reformulation provides the basis for the schematic mode analysis carried out in Sec. V], in which we introduce the crucial zero-frequency modes and describe how they can give rise to a velocity perturbation that behaves as in Eq. (1.2).

In Sec. VI we prepare the way for an actual integration of the perturbation equations by expanding each variable in spherical harmonics. Because the overall perturbation is a composition of an $\ell=1$ rotational perturbation with an $\ell=2$ tidal perturbation, the decomposition involves spherical harmonics with $\ell=1, \ell=2$, and $\ell=3$. These come in two types, even-parity harmonics to represent scalars and vectors, and odd-parity harmonics to represent pseudovectors. The decomposition turns the perturbation equations into three decoupled sets of equations, one set for each value of $\ell$. The explicit integration of these equations is carried out in Sec. VII For concreteness and simplicity we adopt a stellar model based on the polytropic equation of state $p \propto \rho^{2}$, and the solution is obtained with a mixture of analytical and numerical methods. The precise expression of Eq. (1.2) is provided by Eq. (7.6) and the following equations, as well as by Eq. (7.28) and the following equations. The integration reveals that the velocity field includes dipole $(\ell=1)$ and quadrupole $(\ell=2)$ components only; the expected octupole $(\ell=3)$ contribution is absent because of a fortuitous cancellation of the driving force at first post-Newtonian order.

We return to the mode analysis in Sec. VIII] and convert the schematic discussion of Sec. V]into an actual method to solve the perturbation equations for $\delta \boldsymbol{v}$. We confirm that the solution constructed in Sec. VII is indeed generated by a degenerate family of zero-frequency modes, which can be segregated into even-parity $g$-modes (those relevant for the dipole piece of the velocity perturbation) and odd-parity $r$-modes (which are relevant for the quadrupole piece of the perturbation). We therefore validate the suggestion of Ref. [31, that zero-frequency modes are responsible for the gravitomagnetic tidal currents inside a rotating compact body.

A number of additional results are worked out in appendices. In Appendix A we calculate the post-Newtonian approximation to the octupole, rotational-tidal Love number of a $p \propto \rho^{2}$ polytrope. In Appendix B we justify the subtle boundary conditions of the $\ell=1$ perturbation equations at the stellar surface. And finally, in Appendix C we show that the $\ell=1$ acceleration field inside the body averages to a zero overall acceleration for the body's center-of-mass.

\section{GRAVITOMAGNETIC TIDAL FIELD}

Throughout this work we adopt the post-Newtonian approximation to relativistic gravity (see Chapter 8 of Poisson and Will's Gravity [32]), based on the (Newtonian) gravitoelectric potential $U$ and the gravitomagnetic vector potential $U_{a}$. These satisfy the field equations

$$
\nabla^{2} U=-4 \pi G \rho, \quad \nabla^{2} U_{a}=-4 \pi G \rho v_{a},
$$

in which $\rho$ is the mass density of the matter distribution (denoted $\rho^{*}$ in Gravity), and $\boldsymbol{v}$ is its velocity field. The potentials are assumed to satisfy the harmonic gauge condition

$$
\partial_{t} U+\partial_{a} U^{a}=0
$$

and the matter variables satisfy the continuity equation

$$
\partial_{t} \rho+\partial_{a}\left(\rho v^{a}\right)=0 .
$$

A complete description of post-Newtonian gravity also involves an additional potential $\Psi$, which provides a correction of order $(v / c)^{2}$ to the gravitoelectric potential. This potential, however, is not required for our purposes in this work.

We consider a rotating, self-gravitating body of mass $M$, radius $R$, and angular velocity $\Omega$ immersed in a tidal environment created by remote objects. Our considerations are limited to a spherical domain $\mathscr{M}$ described by $0<r<r_{\max }$, where $r$ is the distance to the body's center-of-mass, and $r_{\max }>R$ is a maximum distance from the body. This domain includes the body, but it excludes the remote objects that create the tidal environment.

A Newtonian description of the tidal environment is provided in Sec. 2.5 of Gravity. The gravitoelectric potential is decomposed into a body piece $U^{\text {body }}$ and an external piece $U^{\text {ext }}$, and since the sources of the external potential are outside $\mathscr{M}$, it must satisfy Laplace's equation $\nabla^{2} U^{\text {ext }}=0$. Assuming that the scale of variation of the external potential is large compared with $R$, we express it as the Taylor expansion $U^{\operatorname{ext}}\left(x^{a}\right)=U^{\operatorname{ext}}(0)+g_{a} x^{a}-\frac{1}{2} \mathcal{E}_{a b} x^{a} x^{b}+\cdots$, where $g_{a}:=\partial_{a} U^{\text {ext }}(0)$ and $\mathcal{E}_{a b}:=-\partial_{a b} U^{\text {ext }}(0)$, with $x^{a}$ denoting the position relative to the body's center-of-mass. 
The leading term is an irrelevant constant, the linear term is responsible for the motion of the center-of-mass, and the additional terms are responsible for the tides. To leading order in the tidal interaction, we have that

$$
U^{\text {tidal }}=-\frac{1}{2} \mathcal{E}_{a b} x^{a} x^{b}
$$

with the gravitoelectric tidal quadrupole moment $\mathcal{E}_{a b}(t)$ providing a complete characterization of the tidal environment. The definition of $\mathcal{E}_{a b}$ implies that this tensor is symmetric, and the field equation $\nabla^{2} U^{\text {tidal }}=0$ further implies that it is tracefree: $\mathcal{E}_{a a}=0$. The tidal moment is therefore a symmetric-tracefree (STF) tensor that possesses 5 independent components.

We shall assume that the time scale of variation of $\mathcal{E}_{a b}$ is very long compared with the internal, hydrodynamical time scale of the body, which is comparable to $\sqrt{R^{3} / G M}$. This will allow us to neglect the time derivatives of the tidal gravitoelectric potential.

The gravitomagnetic potential $U_{a}$ also contributes to the tidal environment. We examine the external piece of this potential, which is sourced by the remote objects; it satisfies $\nabla^{2} U_{a}^{\text {ext }}=0$ in addition to the gauge condition $\partial_{a} U_{a}^{\text {ext }}=0$, in which we have neglected the term $\partial_{t} U^{\text {ext }}$, as motivated previously. A gauge transformation $U_{a}^{\text {ext }} \rightarrow U_{a}^{\text {ext }}+\partial_{a} f$ preserves the gauge condition provided that $f$ satisfies Laplace's equation.

We perform a Taylor expansion of the external gravitomagnetic potential, and discard the irrelevant constant term and the linear term responsible for the center-of-mass motion. The tidal potential therefore leads with $U_{a}^{\text {tidal }}=$ $A_{a b c} x^{b} x^{c}$, with $A_{a b c}(t)$ defined to be symmetric in the last two indices; this tensor contains 18 independent components. The gauge condition gives rise to the three constraints $A_{a a b}=0$, and the number of independent components reduces to 15 . An additional reduction is made possible by a gauge transformation generated by $f=C_{a b c} x^{a} x^{b} x^{c}$, where $C_{a b c}$ is completely symmetric by virtue of its definition, and satisfies $C_{a a b}=0$ by virtue of the requirement that $\nabla^{2} f=0$. There are 7 independent components in $C_{a b c}$, and these can be chosen to eliminate an equal number of components in $A_{a b c}$; the count is therefore reduced to 8 independent components. Finally, the field equations $\nabla^{2} U_{a}^{\text {tidal }}=0$ introduce 3 new constraints, and the number of independent components has finally settled to 5 . These can be encoded in the $\mathrm{STF}$ tensor $\mathcal{B}_{a b}(t)$, and it can be verified that

$$
U_{a}^{\text {tidal }}=-\frac{1}{6} \epsilon_{a b c} \mathcal{B}_{d}^{c} x^{b} x^{d}
$$

satisfies the gauge condition (because $\mathcal{B}_{a b}$ is symmetric) and the field equations (because $\mathcal{B}_{a b}$ is tracefree). Equation (2.5), therefore, provides a correct description of a gravitomagnetic tidal potential 33 .

The gravitomagnetic tidal quadrupole moment $\mathcal{B}_{a b}$ can be expressed as $\mathcal{B}_{a b}=2 \epsilon_{c d(a} \partial_{b) c} U_{d}^{\text {ext }}(0)$, in terms of second derivatives of the external potential evaluated at $x^{a}=0$. For a tidal environment created by a single companion of mass $M^{\prime}$ moving with velocity $\boldsymbol{v}^{\prime}$ at a position $\boldsymbol{r}^{\prime}$ from the body, $U_{a}^{\text {ext }}=G M^{\prime} v_{a}^{\prime} / r^{\prime}$, and

$$
\mathcal{B}_{a b}=\frac{6 G M^{\prime}}{r^{\prime 3}}\left(\boldsymbol{n}^{\prime} \times \boldsymbol{v}^{\prime}\right)_{(a} n_{b)}^{\prime}
$$

where $\boldsymbol{n}^{\prime}:=\boldsymbol{r}^{\prime} / r^{\prime}$. When the companion moves on a circular orbit of radius $r^{\prime}$ in the $x-y$ plane of the coordinate system, the nonvanishing components of $\mathcal{B}_{a b}$ are

$$
B_{x z}=\frac{3 G M^{\prime} v^{\prime}}{r^{\prime 3}} \cos \Phi, \quad B_{y z}=\frac{3 G M^{\prime} v^{\prime}}{r^{\prime 3}} \sin \Phi,
$$

where $\Phi:=\omega^{\prime} t$ is the orbital phase, with

$$
\omega^{\prime}:=\sqrt{\frac{G\left(M+M^{\prime}\right)}{r^{\prime 3}}}
$$

denoting the orbital angular velocity, related to the orbital velocity by $v^{\prime}=r^{\prime} \omega^{\prime}$.

\section{BODY'S RESPONSE TO A GRAVITOMAGNETIC TIDAL FIELD}

In this section we derive the equations that govern the response of a rotating body to the tidal gravitomagnetic potential of Eq. 2.5. . We ignore the influence of the gravitoelectric tidal field, which gives rise to the well-understood Newtonian tides (see, for example, Sec. 2.5 of Gravity 32]). The body is modelled as a perfect fluid with a zerotemperature equation of state of the form $p=p(\rho)$, with $p$ denoting the pressure. Its response is determined by the post-Newtonian version of Euler's equation, displayed in Eq. (8.119) of Gravity,

$$
\rho \frac{d v_{a}}{d t}=-\partial_{a} p+\rho \partial_{a} U+\frac{1}{c^{2}}\left[\left(\frac{1}{2} v^{2}+U+\Pi+\frac{p}{\rho}\right) \partial_{a} p-v_{a} \partial_{t} p\right]
$$




$$
+\frac{\rho}{c^{2}}\left[\left(v^{2}-4 U\right) \partial_{a} U-v_{a}\left(3 \partial_{t} U+4 v^{b} \partial_{b} U\right)+4 \partial_{t} U_{a}+4 v^{b}\left(\partial_{b} U_{a}-\partial_{a} U_{b}\right)+\partial_{a} \Psi\right]+O\left(c^{-4}\right),
$$

where $d / d t:=\partial_{t}+v^{b} \partial_{b}$ is the convective time derivative, $\Pi$ is the fluid's internal energy per unit mass, and $\Psi$ is the post-Newtonian potential mentioned previously.

We begin with a nonrotating body in an unperturbed state, in the absence of a perturbing tidal field. In this context the body is static and spherically symmetric, and its structure is determined by the equations $\partial_{a} p=\rho \partial_{a} U+O\left(c^{-2}\right)$ and $\nabla^{2} U=-4 \pi G \rho$. We allow ourselves to neglect all post-Newtonian corrections to the structure equations, which take, in this approximation, the explicit form

$$
\frac{d p}{d r}=\rho \frac{d U}{d r}=-\frac{G m \rho}{r^{2}}, \quad \frac{d m}{d r}=4 \pi r^{2} \rho
$$

with $m(r)$ denoting the internal mass function.

We next switch on the gravitomagnetic tidal field, but keep the body nonrotating. We assume that the tidal field is small and creates a change in the fluid configuration that can adequately be described by a first-order perturbative treatment. We further assume that $\mathcal{B}_{a b}(t)$ changes on a time scale that is long compared with the time scale of internal hydrodynamical processes. And we assume that $\mathcal{B}_{a b}(t \rightarrow-\infty) \rightarrow 0$, so that the body's initial state is the unperturbed state described previously. As we shall see presently, the fluid acquires a velocity field $v_{a}$ as a result of the tidal interaction, and the total gravitomagnetic potential is $U_{a}=U_{a}^{\text {body }}+U_{a}^{\text {tidal }}$, with the body piece satisfying $\nabla^{2} U_{a}^{\text {body }}=-4 \pi G \rho v_{a}$.

The gravitomagnetic tidal perturbation keeps all scalar quantities (such as $\rho, p, U, \Pi$, and $\Psi$ ) unchanged to first order in perturbation theory. The reason is tied to their behavior under a parity transformation, in which $x^{a} \rightarrow-x^{a}$. Scalar quantities are not affected by the transformation, while a vector such as $U_{a}$ changes sign. Now, Eq. 2.5 reveals that $\mathcal{B}_{a b} \rightarrow-\mathcal{B}_{a b}$ under the transformation ( $\epsilon_{a b c}$ is unaffected), and the gravitomagnetic tidal moment therefore behaves as a pseudotensor. Because a perturbation in a scalar quantity would have to be proportional to $\mathcal{B}_{a b} x^{a} x^{b}$ to be a scalar, and because this does change sign under a parity transformation (it is a pseudoscalar instead of a true scalar), we must rule out such perturbations.

With $\delta \rho=\delta p=\delta U=0$, the post-Newtonian Euler equation implies that $\rho d v_{a} / d t=O\left(c^{-2}\right)$, so that the velocity field must be of order $c^{-2}$. This immediately implies that $U_{a}^{\text {body }}=O\left(c^{-2}\right)$, so that

$$
U_{a}=U_{a}^{\text {tidal }}+O\left(c^{-2}\right)=-\frac{1}{6} \epsilon_{a b p} \mathcal{B}_{c}^{p} x^{b} x^{c}+O\left(c^{-2}\right) .
$$

These observations give rise to a huge simplification in Eq. 3.1). A careful examination of the equation, neglecting all terms that are beyond first order in the perturbation, and all terms that are beyond the first post-Newtonian order, reveals that it reduces to $\partial_{t}\left(v_{a}-4 U_{a} / c^{2}\right)=O\left(c^{-4}\right)$. Because the fluid is assumed to be unperturbed initially, the time independence of $v_{a}-4 U_{a} / c^{2}$ guarantees that

$$
v_{a}=\frac{4}{c^{2}} U_{a}+O\left(c^{-4}\right)
$$

at all times. The gravitomagnetic tidal interaction therefore creates a velocity field within the fluid, which gradually builds up as the tidal field is switched on. This velocity field is required by the relativistic circulation theorem [34, 35.

We now allow the body to rotate. For simplicity we take the body to rotate rigidly with an angular velocity $\Omega$. Aligning the rotation axis with the $z$-direction, we define the vector $\Omega^{a}=[0,0, \Omega]$ and the rotational velocity field is

$$
v_{a}^{\mathrm{rot}}=\epsilon_{a b c} \Omega^{b} x^{c} .
$$

We assume that $\Omega$ is sufficiently small that centrifugal effects on the body's structure can be neglected. This amounts to demanding that $\Omega^{2} \ll G m(r) / r^{3}$ throughout the body, and the assumption allows us to work to first order in $v_{a}^{\text {rot }}$. The rotating body is perturbed by the gravitomagnetic tidal field, and the coupling between $v_{a}^{\text {rot }}$ and $U_{a}^{\text {tidal }}$ ensures that in addition to the fluid's velocity field, $\rho, p$, and $U$ also acquire perturbations; parity considerations no longer rule them out, because the pseudovector $v_{a}^{\text {rot }}$ can combine with the pseudotensor $\mathcal{B}_{a b}$ and the vector $x^{a}$ to form scalar quantities. Because the interaction with the gravitomagnetic tidal field is a post-Newtonian effect, all perturbations will be post-Newtonian quantities of order $c^{-2}$.

We let $\rho \rightarrow \rho+\delta \rho, p \rightarrow p+\delta p, U \rightarrow U+\delta U, v_{a} \rightarrow v_{a}^{\text {rot }}+\delta v_{a}$, and $U_{a}=U_{a}^{\text {tidal }}$ in the post-Newtonian Euler equation, and expand the equation to first order in all perturbations, taking into account the important fact that these are all of order $c^{-2}$. After simplification we arrive at

$$
\partial_{t} \delta v_{a}+v^{b} \partial_{b} \delta v_{a}+\left(\partial_{b} v_{a}\right) \delta v^{b}-P_{a}=\frac{4}{c^{2}} W_{a}+O\left(c^{-4}\right)
$$


where

$$
P_{a}:=-\frac{1}{\rho} \partial_{a} \delta p+\frac{\delta \rho}{\rho} \partial_{a} U+\partial_{a} \delta U
$$

and

$$
W_{a}:=\partial_{t} U_{a}+v^{b}\left(\partial_{b} U_{a}-\partial_{a} U_{b}\right) .
$$

To simplify the notation we let $v_{a} \equiv v_{a}^{\text {rot }}$ and $U_{a} \equiv U_{a}^{\text {tidal }}$, as given by Eq. 2.5.

Equation (3.6) is the starting point of the perturbative analysis. Because $\delta v_{a}=4 U_{a} / c^{2}$ when $\Omega=0$, we write

$$
\delta v_{a}=\frac{4}{c^{2}} U_{a}+w_{a}
$$

and consider $w_{a}$ to be a post-Newtonian quantity of order $\Omega$. Making the substitution in Eq. 3.6. and neglecting all terms beyond first order in $\Omega$, we arrive at the simpler equation

$$
\partial_{t} w_{a}-P_{a}=-\frac{4}{c^{2}} A_{a}+O\left(c^{-4}\right),
$$

where

$$
A_{a}:=\left(\partial_{b} v_{a}\right) U^{b}+v^{b} \partial_{a} U_{b} .
$$

The left-hand side of Eq. (3.10) features the familiar linearization of the Newtonian Euler equation for the perturbation of a nonrotating fluid, and the right-hand side features a post-Newtonian driving force $A_{a}$ that originates from the coupling between the gravitomagnetic tidal field $U_{a}$ and the rotational velocity $v_{a}$. The equation is mathematically equivalent to one describing a nonrotating body perturbed by a prescribed driving force.

Equation 3.10 must be supplemented by Poisson's equation

$$
\nabla^{2} \delta U=-4 \pi G \delta \rho
$$

for the perturbation of the Newtonian potential, the continuity equation expressing mass conservation, and an equation of state for the perturbed fluid. Throughout this work we shall assume that the perturbed fluid satisfies the same equation of state as the unperturbed fluid.

\section{LAGRANGIAN DESCRIPTION OF THE FLUID PERTURBATION}

The perturbative treatment of the previous section was couched in the language of Eulerian perturbations, with a perturbation such as $\delta \rho$ comparing the perturbed and unperturbed fluids at the same spatial position. We next introduce a Lagrangian description, in which a perturbation such as $\Delta \rho$ compares the perturbed and unperturbed fluids at the same fluid element. The relation between the two descriptions is provided by the Lagrangian displacement vector $\xi^{a}$, which gives the position of a given fluid element in the perturbed fluid relative to its position in the unperturbed fluid. The Eulerian and Lagrangian perturbations are related by $\Delta=\delta+\xi^{a} \partial_{a}$. In the Lagrangian description, the perturbation in the velocity field is $\Delta v^{a}=d \xi^{a} / d t$, mass conservation is embodied in $\Delta \rho=-\rho \partial_{a} \xi^{a}$, and with the assumption placed earlier on the equation of state, $\Delta p=(d p / d \rho) \Delta \rho$. In terms of Eulerian variations, we have

$$
\delta v^{a}=\partial_{t} \xi^{a}+v^{b} \partial_{b} \xi^{a}-\xi^{b} \partial_{b} v^{a}, \quad \delta \rho=-\partial_{a}\left(\rho \xi^{a}\right),
$$

and $\delta p=(d p / d \rho) \delta \rho$.

We have seen that $\delta v_{a}=4 U_{a} / c^{2}$ when the body is nonrotating $\left(v_{a}=0\right)$. In this case $\delta v_{a}=\partial_{t} \xi^{a}$, and to reflect the change of variables of Eq. (3.9) to account for the rotation, we write

$$
\xi^{a}\left(t, x^{b}\right)=\frac{4}{c^{2}} \int^{t} U^{a}\left(t^{\prime}, x^{b}\right) d t^{\prime}+\zeta^{a}\left(t, x^{b}\right),
$$

where $\zeta^{a}$ is a post-Newtonian quantity of order $\Omega$. Making the substitution in Eq. 4.1 yields

$$
w_{a}\left(t, x^{b}\right)=\partial_{t} \zeta_{a}\left(t, x^{b}\right)+\frac{4}{c^{2}} \int^{t} C_{a}\left(t^{\prime}, x^{b}\right) d t^{\prime}
$$


with

$$
C_{a}:=v^{b} \partial_{b} U_{a}-U^{b} \partial_{b} v_{a}
$$

and

$$
\delta \rho=-\partial_{a}\left(\rho \zeta^{a}\right)
$$

There is no integral term in the last equation, because $\partial_{a} U^{a}=0$ and $U^{a} \partial_{a} \rho=r^{-1}(d \rho / d r) x^{a} U_{a}=0$. The Euler equation 3.10 becomes

$$
\partial_{t t} \zeta_{a}-P_{a}=-\frac{4}{c^{2}} B_{a}+O\left(c^{-4}\right),
$$

with

$$
B_{a}:=v^{b}\left(\partial_{a} U_{b}+\partial_{b} U_{a}\right) .
$$

The equation is again supplemented by Eq. (3.12). The continuity equation has already been incorporated in Eq. 4.5), and as we have seen, the pressure perturbation is given by $\delta p=(d p / d \rho) \delta \rho$.

The integral term in Eq. 4.3) suggests that $w_{a}$ might be expected to grow in time, on a short time scale compared with the scale of variation of the tidal potential. We shall see this expectation confirmed when we construct the solution to the perturbation equations.

\section{MODE ANALYSIS}

Equation 4.6 can be integrated by performing a mode analysis. We examine the homogeneous equation, $\partial_{t t} \zeta_{a}-$ $P_{a}=0$ with $P_{a}$ given by Eq. (3.7), and recognize that by virtue of Eqs. (3.12) and (4.5), $P_{a}$ is a linear functional of $\zeta_{a}$. We express it as $P_{a}=-\mathscr{L}_{a}^{b} \zeta_{b}$, in which $\mathscr{L}_{a}{ }^{b}$ is an integro-differential operator that is known to be self-adjoint with respect to the measure $\rho d^{3} x[36$. Writing

$$
\zeta_{a}\left(t, x^{b}\right)=f_{a}\left(x^{b}\right) e^{-i \omega t},
$$

we find that the homogeneous equation turns into the eigenvalue equation $\mathscr{L}_{a}{ }^{b} f_{b}=\omega^{2} f_{a}$ for the modes $f_{a}$. With $\mathscr{L}_{a}{ }^{b}$ self-adjoint, the eigenvalues $\omega^{2}$ are guaranteed to be real, and modes with different frequencies are guaranteed to be orthogonal. Introducing the mode label $\lambda$, we denote the eigenvalues $\omega_{\lambda}$, the corresponding mode functions $f_{\lambda}^{a}$, and the orthogonality property is expressed by

$$
\int \rho \boldsymbol{f}_{\lambda} \cdot \boldsymbol{f}_{\lambda^{\prime}} d^{3} x=N_{\lambda} \delta_{\lambda \lambda^{\prime}},
$$

with $N_{\lambda}$ denoting the normalization of each mode. The spectrum of $\mathscr{L}_{a}{ }^{b}$ is also known to include an infinitely degenerate set of zero-frequency modes that satisfy $\mathscr{L}_{a}^{b} f_{b}=0$. These are necessarily orthogonal to those with nonzero frequency, and they can be made mutually orthogonal by implementing a Gram-Schmidt procedure. We label the zero-frequency modes with the index $I$, and express their orthogonality as

$$
\int \rho \boldsymbol{f}_{I} \cdot \boldsymbol{f}_{I^{\prime}} d^{3} x=N_{I} \delta_{I I^{\prime}}
$$

We take it for granted that the entire collection of modes $f_{\lambda}^{a}$ and $f_{I}^{a}$ forms a complete set. Completeness under certain assumptions was proved by Beyer and Schmidt [37.

Returning to Eq. (4.6), we decompose $\zeta_{a}$ and $B_{a}$ into modes,

$$
\begin{aligned}
\zeta^{a}\left(t, x^{b}\right) & =\sum_{I} z_{I}(t) f_{I}^{a}\left(x^{b}\right)+\sum_{\lambda} z_{\lambda}(t) f_{\lambda}^{a}\left(x^{b}\right), \\
B^{a}\left(t, x^{b}\right) & =\sum_{I} B_{I}(t) f_{I}^{a}\left(x^{b}\right)+\sum_{\lambda} B_{\lambda}(t) f_{\lambda}^{a}\left(x^{b}\right),
\end{aligned}
$$

with mode amplitudes given by

$$
z_{I}=\frac{1}{N_{I}} \int \rho \boldsymbol{\zeta} \cdot \boldsymbol{f}_{I} d^{3} x, \quad B_{I}=\frac{1}{N_{I}} \int \rho \boldsymbol{B} \cdot \boldsymbol{f}_{I} d^{3} x
$$


and analogous equations for $z_{\lambda}$ and $B_{\lambda}$. We make the substitutions, invoke the mode equation and the orthogonality relations, and obtain

$$
\ddot{z}_{I}=-\frac{4}{c^{2}} B_{I}, \quad \ddot{z}_{\lambda}+\omega_{\lambda}^{2} z_{\lambda}=-\frac{4}{c^{2}} B_{\lambda}
$$

with an overdot indicating differentiation with respect to $t$. Each mode is seen to behave as a driven harmonic oscillator, and with the assumption that the fluid begins in an unperturbed state at $t=-\infty$, the solutions are

$$
z_{I}(t)=-\frac{4}{c^{2}} \int_{-\infty}^{t}\left(t-t^{\prime}\right) B_{I}\left(t^{\prime}\right) d t^{\prime}, \quad z_{\lambda}(t)=-\frac{4}{\omega_{\lambda} c^{2}} \int_{-\infty}^{t} d t^{\prime} B_{\lambda}\left(t^{\prime}\right) \sin \left[\omega_{\lambda}\left(t-t^{\prime}\right)\right] .
$$

While the modes $f_{\lambda}^{a}$ give rise to oscillating contributions to $\zeta_{a}$, the zero-frequency modes $f_{I}^{a}$ produce a growing contribution that can be expressed as

$$
\zeta_{a}^{\text {grow }}\left(t, x^{b}\right)=-\frac{4}{c^{2}} \int_{-\infty}^{t}\left(t-t^{\prime}\right) B_{a}^{\mathrm{zf}}\left(t^{\prime}, x^{b}\right) d t^{\prime}
$$

where $B_{a}^{\mathrm{zf}}\left(t, x^{b}\right):=\sum_{I} B_{I}(t) f_{a}^{I}\left(x^{b}\right)$ is the projection of $B_{a}$, as defined by Eq. (4.7), onto the zero-frequency subspace. This gives rise to a second growing contribution to the velocity field, in addition to the one already displayed in Eq. (4.3). The growing piece of the velocity field is then given by

$$
w_{a}^{\text {grow }}\left(t, x^{b}\right)=\frac{4}{c^{2}} \int^{t}\left[C_{a}\left(t^{\prime}, x^{b}\right)-B_{a}^{\mathrm{zf}}\left(t^{\prime}, x^{b}\right)\right] d t^{\prime},
$$

with $C_{a}$ defined by Eq. (4.4). The zero-frequency modes $f_{I}^{a}$ play a crucial role in the response of a fluid body to a gravitomagnetic tidal field, giving rise to a velocity perturbation that can be expected to grow in time. We shall examine them in detail in Sec. VIII.

\section{SPHERICAL-HARMONIC DECOMPOSITION}

The mode analysis carried out in Sec. V supplies us with a powerful conceptual framework to analyze the perturbation equation (4.6), and it provides us with an expectation that thanks to the zero-frequency modes, the solution $\zeta_{a}$ will contain growing terms. To make further progress we return to Eq. 4.6 and perform a decomposition in spherical harmonics.

To prepare the way for this decomposition we rely on Sec. II of Ref. 38] - see also Sec. II of Ref. 39] - and construct tidal potentials that form an irreducible basis in which to decompose the driving force $B_{a}$ displayed in Eq. 4.7). We first introduce the spherical coordinates $\left(r, \theta^{A}\right)$, with $\theta^{A}=(\theta$, $\phi)$, which are related to the Cartesian coordinates by $x^{a}=r n^{a}$, with $n^{a}:=[\sin \theta \cos \phi, \sin \theta \sin \phi, \cos \theta]$. We also introduce the spherical-harmonic functions $Y^{\ell \mathrm{m}}\left(\theta^{A}\right)$ displayed in Table I these are defined to be real functions, they are not normalized in the usual way, and the label $\mathrm{m}$ describes their dependence on $\phi$. The association

$$
\mathcal{B}_{a b} n^{a} n^{b}=\sum_{\mathrm{m}} \mathcal{B}_{\mathrm{m}}^{\mathrm{q}} Y^{2, \mathrm{~m}}
$$

allows us to package the five independent components of $\mathcal{B}_{a b}$ into the five harmonic coefficients $\mathcal{B}_{\mathrm{m}}^{\mathrm{q}}$. The superscript q stands for "quadrupole", and the explicit relations between $\mathcal{B}_{a b}$ and $\mathcal{B}_{\mathrm{m}}^{\mathrm{q}}$ are listed in Table II.

The pseudovector $\Omega_{a}$ and pseudotensor $\mathcal{B}_{a b}$ can be combined to form the vector and symmetric-tracefree (STF) tensor

$$
\mathcal{K}_{a}:=\mathcal{B}_{a b} \Omega^{b}, \quad \mathcal{K}_{a b c}:=\mathcal{B}_{\langle a b} \Omega_{c\rangle},
$$

in which the angular brackets instruct us to symmetrize all indices and remove all traces. They can also be combined into the STF pseudotensor

$$
\hat{\mathcal{B}}_{a b}:=2 \Omega^{c} \epsilon_{c d(a} \mathcal{B}^{d}{ }_{b)}
$$

The associations

$$
\mathcal{K}_{a} n^{a}=\sum_{\mathrm{m}} \mathcal{K}_{\mathrm{m}}^{\mathrm{d}} Y^{1, \mathrm{~m}}, \quad \mathcal{K}_{a b c} n^{a} n^{b} n^{c}=\sum_{\mathrm{m}} \mathcal{K}_{\mathrm{m}}^{\mathrm{o}} Y^{3, \mathrm{~m}}, \quad \hat{\mathcal{B}}_{a b} n^{a} n^{b}=\sum_{\mathrm{m}} \hat{\mathcal{B}}_{\mathrm{m}}^{\mathrm{q}} Y^{2, \mathrm{~m}}
$$


TABLE I. Spherical-harmonic functions $Y^{\ell m}$. The functions are real, and they are listed for the relevant modes $l=1$ (dipole), $l=2$ (quadrupole), and $l=3$ (octupole). The abstract index $\mathrm{m}$ describes the dependence of these functions on the angle $\phi$; for example $Y^{\ell, 2 s}$ is proportional to $\sin 2 \phi$.

$$
\begin{aligned}
& Y^{1,0}=\cos \theta \\
& Y^{1,1 c}=\sin \theta \cos \phi \\
& Y^{1,1 s}=\sin \theta \sin \phi \\
& Y^{2,0}=1-3 \cos ^{2} \theta \\
& Y^{2,1 c}=2 \sin \theta \cos \theta \cos \phi \\
& Y^{2,1 s}=2 \sin \theta \cos \theta \sin \phi \\
& Y^{2,2 c}=\sin ^{2} \theta \cos 2 \phi \\
& Y^{2,2 s}=\sin ^{2} \theta \sin 2 \phi \\
& Y^{3,0}=\cos \theta\left(3-5 \cos ^{2} \theta\right) \\
& Y^{3,1 c}=\frac{3}{2} \sin \theta\left(1-5 \cos ^{2} \theta\right) \cos \phi \\
& Y^{3,1 s}=\frac{3}{2} \sin \theta\left(1-5 \cos ^{2} \theta\right) \sin \phi \\
& Y^{3,2 c}=3 \sin ^{2} \theta \cos \theta \cos _{2} \phi \\
& Y^{3,2 s}=3 \sin ^{2} \theta \cos \theta \sin 2 \phi \\
& Y^{3,3 c}=\sin ^{3} \theta \cos 3 \phi \\
& Y^{3,3 s}=\sin ^{3} \theta \sin 3 \phi
\end{aligned}
$$

TABLE II. Spherical-harmonic coefficients of tidal potentials.

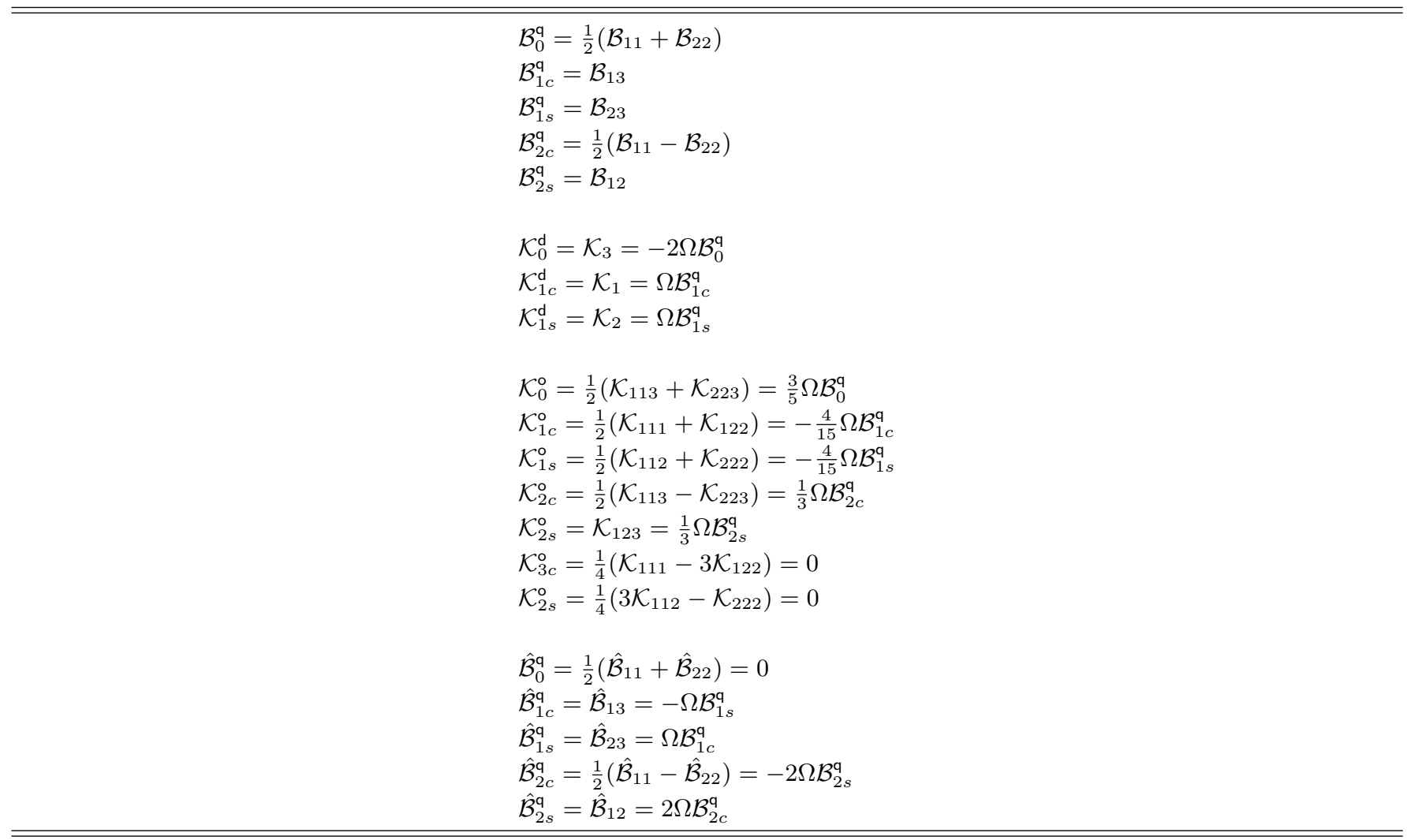


define the harmonic coefficients $\mathcal{K}_{\mathrm{m}}^{\mathrm{d}}, \mathcal{K}_{\mathrm{m}}^{\circ}$, and $\hat{\mathcal{B}}_{\mathrm{m}}^{\mathrm{q}}$, which are given explicitly in Table II. We may note that

$$
\mathcal{K}_{\mathrm{m}}^{\mathrm{d}}=\lambda_{\mathrm{m}}^{\mathrm{d}} \Omega \mathcal{B}_{\mathrm{m}}^{\mathrm{q}}, \quad \mathcal{K}_{\mathrm{m}}^{\circ}=\lambda_{\mathrm{m}}^{\circ} \Omega \mathcal{B}_{\mathrm{m}}^{\mathrm{q}},
$$

where the numbers $\lambda_{\mathrm{m}}^{\mathrm{d}}$ and $\lambda_{\mathrm{m}}^{\circ}$ can be extracted from the table. The superscripts $\mathrm{d}$ and o stand for "dipole" and "octupole", respectively.

The tidal potentials are divided into scalar and vector potentials. For our purposes here, a "scalar" is a quantity that stays invariant under a transformation of the angular coordinates $\theta^{A}$, while a "vector" is a quantity that transforms as a one-form under this transformation. The scalar potentials can be decomposed into scalar harmonics $Y^{\ell m}$, but the vector potentials require the even-parity vector harmonics

$$
Y_{A}^{\ell m}:=\partial_{A} Y^{\ell m}
$$

and the odd-parity vector harmonics

$$
X_{A}^{\ell m}:=-\epsilon_{A}^{B} \partial_{B} Y^{\ell m}
$$

in which $\epsilon_{A}^{B}$ is the Levi-Civita tensor on the unit 2-sphere, with nonvanishing components $\epsilon_{\theta}^{\phi}=1 / \sin \theta$ and $\epsilon_{\phi}^{\theta}=$ $-\sin \theta$.

We may now state the definition of the tidal potentials. They are given by

$$
\begin{aligned}
\mathcal{K}^{\mathrm{d}}:=\sum_{\mathrm{m}} \mathcal{K}_{\mathrm{m}}^{\mathrm{d}} Y^{1, \mathrm{~m}}, \quad \mathcal{K}_{A}^{\mathrm{d}}:=\sum_{\mathrm{m}} \mathcal{K}_{\mathrm{m}}^{\mathrm{d}} Y_{A}^{1, \mathrm{~m}}, \\
\mathcal{K}^{\circ}:=\sum_{\mathrm{m}} \mathcal{K}_{\mathrm{m}}^{\circ} Y^{3, \mathrm{~m}}, \quad \mathcal{K}_{A}^{\circ}:=\frac{1}{3} \sum_{\mathrm{m}} \mathcal{K}_{\mathrm{m}}^{\circ} Y_{A}^{3, \mathrm{~m}}, \\
\hat{\mathcal{B}}_{A}^{\mathrm{q}}:=\frac{1}{2} \sum_{\mathrm{m}} \hat{\mathcal{B}}_{\mathrm{m}}^{\mathrm{q}} X_{A}^{2, \mathrm{~m}}=-\frac{1}{2} \Omega \sum_{\mathrm{m}} \mathcal{B}_{\mathrm{m}}^{\mathrm{q}} \partial_{\phi} X_{A}^{2, \mathrm{~m}},
\end{aligned}
$$

and they can be used as a basis to decompose the driving force $B_{a}$ displayed in Eq. (4.7). Simple manipulations reveal that

$$
\begin{aligned}
& B_{r}:=B_{a} n^{a}=\frac{1}{10} r^{2} \mathcal{K}^{\mathrm{d}}-\frac{1}{6} r^{2} \mathcal{K}^{\circ}, \\
& B_{A}:=r B_{a} \partial_{A} n^{a}=\frac{1}{5} r^{3} \mathcal{K}_{A}^{\mathrm{d}}-\frac{1}{6} r^{3} \mathcal{K}_{A}^{\circ}+\frac{1}{9} r^{3} \hat{\mathcal{B}}_{A}^{\mathrm{q}} .
\end{aligned}
$$

The vector $C_{a}$ defined by Eq. (4.4) can also be decomposed in this basis. Here we find that

$$
C_{r}:=C_{a} n^{a}=0, \quad C_{A}:=r C_{a} \partial_{A} n^{a}=\frac{1}{6} r^{3} \hat{\mathcal{B}}_{A}^{q} .
$$

The fact that $B_{r}$ can be decomposed in spherical harmonics with $\ell=1$ and $\ell=3$ implies that all scalar perturbations can be decomposed in a similar way. And the fact that $B_{A}$ can be decomposed in even-parity vector harmonics with $\ell=(1,3)$ and in odd-parity harmonics with $\ell=2$ ensures that all vector perturbations can be decomposed in the same way. We therefore write

$$
\begin{aligned}
\delta p & =\Omega \sum_{\mathrm{m}} \lambda_{\mathrm{m}}^{\mathrm{d}} p^{\mathrm{d}}(t, r) Y^{1, \mathrm{~m}}+\Omega \sum_{\mathrm{m}} \lambda_{\mathrm{m}}^{\circ} p^{\mathrm{o}}(t, r) Y^{3, \mathrm{~m}}, \\
\delta U & =\Omega \sum_{\mathrm{m}} \lambda_{\mathrm{m}}^{\mathrm{d}} U^{\mathrm{d}}(t, r) Y^{1, \mathrm{~m}}+\Omega \sum_{\mathrm{m}} \lambda_{\mathrm{m}}^{\circ} U^{\circ}(t, r) Y^{3, \mathrm{~m}}, \\
\zeta_{r} & =\Omega \sum_{\mathrm{m}} \lambda_{\mathrm{m}}^{\mathrm{d}} \zeta_{r}^{\mathrm{d}}(t, r) Y^{1, \mathrm{~m}}+\Omega \sum_{\mathrm{m}} \lambda_{\mathrm{m}}^{\circ} \zeta_{r}^{\circ}(t, r) Y^{3, \mathrm{~m}}, \\
w_{r} & =\Omega \sum_{\mathrm{m}} \lambda_{\mathrm{m}}^{\mathrm{d}} w_{r}^{\mathrm{d}}(t, r) Y^{1, \mathrm{~m}}+\Omega \sum_{\mathrm{m}} \lambda_{\mathrm{m}}^{\circ} w_{r}^{o}(t, r) Y^{3, \mathrm{~m}}
\end{aligned}
$$

as well as

$$
\zeta_{A}=\Omega \sum_{\mathrm{m}} \lambda_{\mathrm{m}}^{\mathrm{d}} \zeta^{\mathrm{d}}(t, r) Y_{A}^{1, \mathrm{~m}}+\frac{1}{3} \Omega \sum_{\mathrm{m}} \lambda_{\mathrm{m}}^{\circ} \zeta^{\circ}(t, r) Y_{A}^{3, \mathrm{~m}}-\frac{1}{2} \Omega \sum_{\mathrm{m}} \hat{\zeta}^{\mathrm{q}}(t, r) \partial_{\phi} X_{A}^{2, \mathrm{~m}},
$$




$$
w_{A}=\Omega \sum_{\mathrm{m}} \lambda_{\mathrm{m}}^{\mathrm{d}} w^{\mathrm{d}}(t, r) Y_{A}^{1, \mathrm{~m}}+\frac{1}{3} \Omega \sum_{\mathrm{m}} \lambda_{\mathrm{m}}^{\mathrm{o}} w^{\mathrm{o}}(t, r) Y_{A}^{3, \mathrm{~m}}-\frac{1}{2} \Omega \sum_{\mathrm{m}} \hat{w}^{\mathrm{q}}(t, r) \partial_{\phi} X_{A}^{2, \mathrm{~m}}
$$

A decomposition for $\delta \rho$ is not required, because the equation of state provides a direct relation to $\delta p$. Factors of $\lambda_{\mathrm{m}}^{\mathrm{d}}$ and $\lambda_{\mathrm{m}}^{\circ}$ are inserted within the sums over $\mathrm{m}$ to simplify the resulting equations, and $\mathrm{m}$ labels on the various coefficients $p^{\mathrm{d}}, \cdots, \hat{w}^{\mathrm{q}}$ are omitted to keep the notation uncluttered. As we shall see, the perturbation equations satisfied by these quantities will all be independent of $\mathrm{m}$, except for the driving terms involving the gravitomagnetic tidal moments $\mathcal{B}_{\mathrm{m}}^{\mathrm{q}}$. The infrastructure put in place here, elaborate though it may seem, produces a substantial simplification of the resulting perturbation equations.

We next transform Eqs. 4.3), 4.5, and (4.6) from the Cartesian coordinates $x^{a}$ to the spherical coordinates $\left(r, \theta^{A}\right)$, and substitute the decompositions in spherical harmonics. This returns a large set of equations, with subsets that decouple from one another. In the dipole sector we have

$$
\begin{aligned}
& 0=\partial_{t t} \zeta_{r}^{\mathrm{d}}+\partial_{r}\left(p^{\mathrm{d}} / \rho-U^{\mathrm{d}}\right)+\frac{2}{5 c^{2}} r^{2} \mathcal{B}_{\mathrm{m}}^{\mathrm{q}}(t), \\
& 0=\partial_{t t} \zeta^{\mathrm{d}}+p^{\mathrm{d}} / \rho-U^{\mathrm{d}}+\frac{4}{5 c^{2}} r^{3} \mathcal{B}_{\mathrm{m}}^{\mathrm{q}}(t), \\
& 0=\rho \partial_{r} \zeta_{r}^{\mathrm{d}}+\left(\rho^{\prime}+2 \rho / r\right) \zeta_{r}^{\mathrm{d}}-\frac{2 \rho}{r^{2}} \zeta^{\mathrm{d}}-\frac{\rho^{\prime} r^{2}}{G m}\left(p^{\mathrm{d}} / \rho\right), \\
& 0=\partial_{r r} U^{\mathrm{d}}+\frac{2}{r} \partial_{r} U^{\mathrm{d}}-\frac{2}{r^{2}} U^{\mathrm{d}}-\frac{4 \pi r^{2} \rho^{\prime}}{m}\left(p^{\mathrm{d}} / \rho\right), \\
& w_{r}^{\mathrm{d}}=\partial_{t} \zeta_{r}^{\mathrm{d}}, \quad w^{\mathrm{d}}=\partial_{t} \zeta^{\mathrm{d}},
\end{aligned}
$$

where $\rho^{\prime}:=d \rho / d r$. In the octupole sector we have

$$
\begin{aligned}
& 0=\partial_{t t} \zeta_{r}^{\circ}+\partial_{r}\left(p^{\circ} / \rho-U^{\circ}\right)-\frac{2}{3 c^{2}} r^{2} \mathcal{B}_{\mathrm{m}}^{\mathrm{q}}(t), \\
& 0=\partial_{t t} \zeta^{\circ}+3\left(p^{\circ} / \rho-U^{\circ}\right)-\frac{2}{3 c^{2}} r^{3} \mathcal{B}_{\mathrm{m}}^{\mathrm{q}}(t), \\
& 0=\rho \partial_{r} \zeta_{r}^{\circ}+\left(\rho^{\prime}+2 \rho / r\right) \zeta_{r}^{\circ}-\frac{4 \rho}{r^{2}} \zeta^{\circ}-\frac{\rho^{\prime} r^{2}}{G m}\left(p^{\circ} / \rho\right), \\
& 0=\partial_{r r} U^{\circ}+\frac{2}{r} \partial_{r} U^{\circ}-\frac{12}{r^{2}} U^{\circ}-\frac{4 \pi r^{2} \rho^{\prime}}{m}\left(p^{\circ} / \rho\right), \\
& w_{r}^{\circ}=\partial_{t} \zeta_{r}^{\circ}, \quad w^{\circ}=\partial_{t} \zeta^{\circ},
\end{aligned}
$$

and the quadrupole sector is limited to

$$
\begin{aligned}
& 0=\partial_{t t} \hat{\zeta}^{\mathrm{q}}+\frac{4}{9 c^{2}} r^{3} \mathcal{B}_{\mathrm{m}}^{\mathrm{q}}(t) \\
& \hat{w}^{\mathrm{q}}=\partial_{t} \hat{\zeta}^{\mathrm{q}}+\frac{2}{3 c^{2}} r^{3} \int^{t} \mathcal{B}_{\mathrm{m}}^{\mathrm{q}}\left(t^{\prime}\right) d t^{\prime}
\end{aligned}
$$

\section{SOLUTION TO THE PERTURBATION EQUATIONS}

In this section we integrate the perturbation equations displayed in Eqs. (6.13), 6.14), and (6.15). For concreteness and simplicity we choose a stellar model corresponding to the polytropic equation of state $p=K \rho^{2}$, where $K$ is a constant. The structure equations for this model return

$$
\rho=\frac{M}{4 R^{2} r} \sin (\pi r / R), \quad m=\frac{M}{\pi}\left[\sin (\pi r / R)-\frac{\pi r}{R} \cos (\pi r / R)\right]
$$

for the density and mass functions, respectively, and

$$
p=\frac{G M^{2}}{8 \pi R^{2} r^{2}} \sin ^{2}(\pi r / R)
$$

for the pressure. The equations also return $K=2 G R^{2} / \pi$ for the constant appearing in the equation of state. 


\section{A. Quadrupole sector}

The solution to Eq. 6.15a is immediate, and actually independent of the equation of state:

$$
\hat{\zeta}^{\mathrm{q}}=-\frac{4}{9 c^{2}} r^{3} \int_{-\infty}^{t}\left(t-t^{\prime}\right) \mathcal{B}_{\mathrm{m}}^{\mathrm{q}}\left(t^{\prime}\right) d t^{\prime}
$$

Equation $6.15 \mathrm{~b}$ then gives

$$
\hat{w}^{\mathrm{q}}=\frac{2}{9 c^{2}} r^{3} \int_{-\infty}^{t} \mathcal{B}_{\mathrm{m}}^{\mathrm{q}}\left(t^{\prime}\right) d t^{\prime},
$$

and this represents a growing contribution to the velocity field. Substituting this within Eq. 6.12b and recalling Eq. $6.8 \mathrm{c}$, we find that the quadrupole term in the velocity perturbation is given by

$$
w_{A}^{\ell=2}=\frac{2}{9 c^{2}} r^{3} \int_{-\infty}^{t} \hat{\mathcal{B}}_{A}^{q}\left(t^{\prime}\right) d t^{\prime} ;
$$

the radial component of the velocity field vanishes.

The velocity perturbation becomes

$$
w_{a}^{\ell=2}=\frac{2}{9 c^{2}} \epsilon_{a b c} x^{b} x^{d} \int_{-\infty}^{t} \hat{\mathcal{B}}_{d}^{c}\left(t^{\prime}\right) d t^{\prime}
$$

after conversion to Cartesian coordinates, with $\hat{\mathcal{B}}_{a b}$ defined by Eq. 6.3). For the specific tidal environment described by Eq. (2.7) and corresponding to a companion body of mass $M^{\prime}$ moving on a circular orbit of radius $r^{\prime}$ in the body's equatorial plane, the quadrupole velocity field is

$$
\begin{aligned}
& w_{x}^{\ell=2}=-\frac{G M^{\prime} v^{\prime} R^{2}}{3 c^{2} r^{\prime 3}} \frac{\Omega}{\omega^{\prime}}\left[\left(3 \cos ^{2} \theta-1\right) \sin \Phi+\sin ^{2} \theta \sin (\Phi-2 \phi)\right] \bar{r}^{2}, \\
& w_{y}^{\ell=2}=\frac{G M^{\prime} v^{\prime} R^{2}}{3 c^{2} r^{\prime}} \frac{\Omega}{\omega^{\prime}}\left[\left(3 \cos ^{2} \theta-1\right) \cos \Phi-\sin ^{2} \theta \cos (\Phi-2 \phi)\right] \bar{r}^{2}, \\
& w_{z}^{\ell=2}=\frac{2 G M^{\prime} v^{\prime} R^{2}}{3 c^{2} r^{\prime 3}} \frac{\Omega}{\omega^{\prime}} \sin \theta \cos \theta \sin (\Phi-\phi) \bar{r}^{2},
\end{aligned}
$$

where $v^{\prime}=r^{\prime} \omega^{\prime}$ is the orbital velocity, $\omega^{\prime}$ the orbital angular velocity of Eq. 2.8), $\Phi:=\omega^{\prime} t$ the orbital phase, $(\theta, \phi)$ the polar angles associated with the coordinates $x^{a}$, and $\bar{r}:=r / R$. To arrive at these expressions it was assumed that $r^{\prime}$ varies over a radiation-reaction time scale that is much longer than $1 / \omega^{\prime}$, and that $r^{\prime}=\infty$ at $t=-\infty$; this is consistent with our previous assumption that the body begins in an unperturbed state.

\section{B. Octupole sector}

Combining Eqs. 6.14a and 6.14b yields $\partial_{t t}\left(\zeta_{r}^{\circ}-\frac{1}{3} \partial_{r} \zeta^{\circ}\right)=0$, with the terms involving the driving force cancelling out. The vanishing initial conditions at $t=-\infty$ imply that $\zeta_{r}^{\circ}-\frac{1}{3} \partial_{r} \zeta^{\circ}=0$ at all times, and this combination of perturbation quantities is therefore unable to grow in time. While $\zeta_{r}^{\mathrm{o}}$ and $\zeta^{\circ}$ could grow individually, we assume that this does not occur. In view of Eq. (5.8), this amounts to an assumption that the $\ell=3$ piece of $B_{a}^{\text {zf }}$ actually vanishes, which prevents the growth of the octupole piece of the Lagrangian displacement vector. This assumption will be justified in Sec. VIII

To reflect this assumption we make the ansatz

$$
\zeta_{r}^{\mathrm{o}}=\mathrm{y}_{r}^{\mathrm{o}}(r) \mathcal{B}_{\mathrm{m}}^{\mathrm{q}}(t), \quad \zeta^{\circ}=\mathrm{y}^{\circ}(r) \mathcal{B}_{\mathrm{m}}^{\mathrm{q}}(t)
$$

for the Lagrangian displacement, with $\mathrm{y}_{r}^{\circ}=\frac{1}{3} d \mathrm{y}^{\circ} / d r$, and we write

$$
p^{\circ}=\mathrm{p}^{\circ}(r) \mathcal{B}_{\mathrm{m}}^{\mathrm{q}}(t), \quad U^{\circ}=\mathrm{U}^{\circ}(r) \mathcal{B}_{\mathrm{m}}^{\mathrm{q}}(t)
$$

for the remaining perturbations. We neglect the time derivatives when we make the substitutions into the perturbation equations. Equation $6.14 \mathrm{~b}$ then produces

$$
\mathrm{p}^{\circ} / \rho=\mathrm{U}^{\circ}+\frac{2}{9 c^{2}} r^{3}
$$


and inserting this in Eq. 6.14d yields

$$
\frac{d^{2} \mathrm{U}^{\circ}}{d r^{2}}+\frac{2}{r} \frac{d \mathrm{U}^{\circ}}{d r}-\left(\frac{12}{r^{2}}+\frac{4 \pi r^{2} \rho^{\prime}}{m}\right) \mathrm{U}^{\circ}=\frac{2}{9 c^{2}} \frac{4 \pi r^{5} \rho^{\prime}}{m} .
$$

Equation $6.14 \mathrm{c}$ gives rise to a second-order differential equation for $\mathrm{y}^{\circ}$, which we shall not concern ourselves with, since the physical aspects of the perturbation are completely captured by Eqs. (7.10) and (7.11).

Equation (7.11) can be solved analytically for the polytropic model introduced previously. We require the solution to be regular at $r=0$, and to match smoothly with an external solution of the form $\mathrm{U}^{\circ} \propto r^{-4}$ at $r=R$. This solution is given by

$$
\mathrm{U}^{\circ}=-\frac{2}{9 c^{2}} r^{3}\left[1+\frac{7 R^{5}}{\pi^{3} r^{7}}\left(2 \pi^{2} r^{2}-5 R^{2}\right) \sin (\pi r / R)-\frac{7 R^{4}}{3 \pi^{2} r^{6}}\left(\pi^{2} r^{2}-15 R^{2}\right) \cos (\pi r / R)\right] .
$$

The function within square brackets behaves as $1-\pi^{4} / 45+O\left(r^{2} / R^{2}\right)$ close to $r=0$, and as $5\left(2 \pi^{2}-21\right) /\left(3 \pi^{2}\right)+$ $O(1-r / R)$ close to $r=R$. Equation 7.10 then gives

$$
\mathrm{p}^{\circ} / \rho=-\frac{2}{9 c^{2}} r^{3}\left[\frac{7 R^{5}}{\pi^{3} r^{7}}\left(2 \pi^{2} r^{2}-5 R^{2}\right) \sin (\pi r / R)-\frac{7 R^{4}}{3 \pi^{2} r^{6}}\left(\pi^{2} r^{2}-15 R^{2}\right) \cos (\pi r / R)\right] .
$$

In this expression, the function within square brackets behaves as $-\pi^{4} / 45+O\left(r^{2} / R^{2}\right)$ close to $r=0$, and as $7\left(\pi^{2}-\right.$ $15) /\left(3 \pi^{2}\right)+O(1-r / R)$ close to $r=R$.

It can be observed that $\mathrm{p}^{\circ} / \rho$ approaches a nonzero value at $r=R$; with $\rho(R)=0$, this means that $\mathrm{p}^{\circ}$ itself vanishes at the stellar surface. The correct surface condition can be inferred from $\Delta \rho=0$ at $r=R$, which implies $\delta \rho+\rho^{\prime} \zeta_{r}=0$. On the other hand, the equation of state and the structure equations imply $\delta p / \rho=-\left(G m / r^{2}\right) \delta \rho / \rho^{\prime}$, and combining these equations yields

$$
\left.\frac{\delta p}{\rho}\right|_{r=R}=\frac{G M}{R^{2}} \zeta_{r}(r=R)
$$

Because $\zeta_{r} \neq 0$ at the surface, it follows that $\delta p / \rho$ must be nonvanishing as well.

Inserting Eqs. (7.12) and $(7.13)$ into Eqs. (6.11b) and (6.11a), respectively, and recalling Eqs. (6.4), (6.5), and (6.8b), we find that the octupole piece of the potential and pressure perturbations are given by

$$
\delta U^{\ell=3}=-\frac{2}{9 c^{2}} \mathcal{K}_{a b c} x^{a} x^{b} x^{c}\left[1+\frac{7 R^{5}}{\pi^{3} r^{7}}\left(2 \pi^{2} r^{2}-5 R^{2}\right) \sin (\pi r / R)-\frac{7 R^{4}}{3 \pi^{2} r^{6}}\left(\pi^{2} r^{2}-15 R^{2}\right) \cos (\pi r / R)\right]
$$

and

$$
\delta p^{\ell=3} / \rho=-\frac{2}{9 c^{2}} \mathcal{K}_{a b c} x^{a} x^{b} x^{c}\left[\frac{7 R^{5}}{\pi^{3} r^{7}}\left(2 \pi^{2} r^{2}-5 R^{2}\right) \sin (\pi r / R)-\frac{7 R^{4}}{3 \pi^{2} r^{6}}\left(\pi^{2} r^{2}-15 R^{2}\right) \cos (\pi r / R)\right],
$$

where $\mathcal{K}_{a b c}$ is defined by Eq. 6.2).

Our expression for $\delta U^{\ell=3}$ is used in Appendix A to calculate the rotational-tidal Love number of the polytropic stellar model.

\section{Dipole sector}

Combining Eqs. 6.13a and 6.13b gives

$$
\partial_{t t}\left(\zeta_{r}^{\mathrm{d}}-\partial_{r} \zeta^{\mathrm{d}}\right)=\frac{2}{c^{2}} r^{2} \mathcal{B}_{\mathrm{m}}^{\mathrm{q}}(t)
$$

and in this case we see a nonzero driving force on the right-hand side of the equation. This implies that $\zeta_{r}^{\mathrm{d}}-\partial_{r} \zeta^{\mathrm{d}}$ must grow with time, and to proceed we assume that $\zeta_{r}^{\mathrm{d}}$ and $\zeta^{\mathrm{d}}$ grow individually. To reflect this we make the ansatz

$$
\zeta_{r}^{\mathrm{d}}=\mathrm{z}_{r}^{\mathrm{d}}(r) \int_{-\infty}^{t}\left(t-t^{\prime}\right) \mathcal{B}_{\mathrm{m}}^{\mathrm{q}}\left(t^{\prime}\right) d t^{\prime}+\mathrm{y}_{r}^{\mathrm{d}}(r) \mathcal{B}_{\mathrm{m}}^{\mathrm{q}}(t)
$$




$$
\zeta_{r}=\mathrm{z}^{\mathrm{d}}(r) \int_{-\infty}^{t}\left(t-t^{\prime}\right) \mathcal{B}_{\mathrm{m}}^{\mathrm{q}}\left(t^{\prime}\right) d t^{\prime}+\mathrm{y}^{\mathrm{d}}(r) \mathcal{B}_{\mathrm{m}}^{\mathrm{q}}(t)
$$

for the Lagrangian displacement vector, and

$$
p^{\mathrm{d}}=\mathrm{p}^{\mathrm{d}}(r) \mathcal{B}_{\mathrm{m}}^{\mathrm{q}}(t), \quad U^{\mathrm{d}}=\mathrm{U}^{\mathrm{d}}(r) \mathcal{B}_{\mathrm{m}}^{\mathrm{q}}(t)
$$

for the remaining perturbations. The assumption leading to Eq. (7.18) will be justified in Sec. VIII, where we show that the dipole piece of $B_{a}^{\mathrm{zf}}$ does not vanish and therefore leads to a growing displacement vector.

We insert the preceding equations into Eqs. (6.13), neglect terms involving second derivatives of $\mathcal{B}_{\mathrm{m}}^{\mathrm{q}}(t)$, and obtain the system of equations

$$
\begin{aligned}
& 0=z_{r}^{\mathrm{d}}-\frac{d \mathrm{z}^{\mathrm{d}}}{d r}-\frac{2}{c^{2}} r^{2}, \\
& 0=\mathrm{z}^{\mathrm{d}}+\mathrm{p}^{\mathrm{d}} / \rho-\mathrm{U}^{\mathrm{d}}+\frac{4}{5 c^{2}} r^{3}, \\
& 0=\rho \frac{d \mathrm{z}_{r}^{\mathrm{d}}}{d r}+\left(\rho^{\prime}+2 \rho / r\right) \mathrm{z}_{r}^{\mathrm{d}}-\frac{2 \rho}{r^{2}} \mathrm{z}^{\mathrm{d}}, \\
& 0=\frac{d^{2} \mathrm{U}^{\mathrm{d}}}{d r^{2}}+\frac{2}{r} \frac{d \mathrm{U}^{\mathrm{d}}}{d r}-\frac{2}{r} \mathrm{U}^{\mathrm{d}}-\frac{4 \pi r^{2} \rho^{\prime}}{m}\left(\mathrm{p}^{\mathrm{d}} / \rho\right)
\end{aligned}
$$

for the radial functions $\mathrm{z}_{r}^{\mathrm{d}}, \mathrm{z}^{\mathrm{d}}, \mathrm{p}^{\mathrm{d}}$, and $\mathrm{U}^{\mathrm{d}}$. An equation can also derived for $\mathrm{y}_{r}^{\mathrm{o}}$ and $\mathrm{y}^{\mathrm{o}}$, but these variables are of no concern to us.

Equation $7.20 \mathrm{~b}$ allows us to eliminate $z^{\mathrm{d}}$ from the system, and substitution into Eqs. 7.20a and 7.20c produces

$$
\begin{aligned}
& 0=r \frac{d \mathrm{z}_{r}^{\mathrm{d}}}{d r}+\frac{r \rho^{\prime}+2 \rho}{\rho} \mathrm{z}_{r}^{\mathrm{d}}+\frac{2}{r}\left(\mathrm{p}^{\mathrm{d}} / \rho-\mathrm{U}^{\mathrm{d}}\right)+\frac{8}{5 c^{2}} r^{2}, \\
& 0=r \frac{d}{d r}\left(\mathrm{p}^{\mathrm{d}} / \rho\right)+r \mathrm{z}_{r}^{\mathrm{d}}-r \frac{d \mathrm{U}^{\mathrm{d}}}{d r}+\frac{2}{5 c^{2}} r^{3} .
\end{aligned}
$$

These equations, together with Eq. $7.20 \mathrm{~d}$, form a closed set of equations for $z_{r}^{\mathrm{d}}, \mathrm{p}^{\mathrm{d}}$, and $\mathrm{U}^{\mathrm{d}}$. These must be integrated numerically.

To facilitate the numerical work we introduce the new variables $e_{1}, \cdots, e_{4}$ defined by

$$
\mathrm{z}_{r}^{\mathrm{d}}=\frac{R^{2}}{c^{2}} e_{1}, \quad \mathrm{p}^{\mathrm{d}} / \rho=\frac{R^{2}}{c^{2}} r e_{2}, \quad \mathrm{U}^{\mathrm{d}}=\frac{R^{2}}{c^{2}} r e_{3}, \quad \frac{d \mathrm{U}^{\mathrm{d}}}{d r}=\frac{R^{2}}{c^{2}} e_{4} .
$$

We further define the dimensionless radial variable $\bar{r}:=r / R$, density function $\bar{\rho}:=\left(R^{3} / M\right) \rho$, and mass function $\bar{m}:=m / M$. In terms of all this we have that

$$
\mathrm{z}^{\mathrm{d}}=\frac{R^{2}}{c^{2}} r e_{5}, \quad e_{5}:=-e_{2}+e_{3}-\frac{4}{5} \bar{r}^{2},
$$

and the perturbation equations become

$$
\begin{aligned}
& \bar{r} e_{1}^{\prime}=-\frac{\bar{r} \bar{\rho}^{\prime}+2 \bar{\rho}}{\bar{\rho}} e_{1}-2 e_{2}+2 e_{3}-\frac{8}{5} \bar{r}^{2}, \\
& \bar{r} e_{2}^{\prime}=-e_{1}-e_{2}+e_{4}-\frac{2}{5} \bar{r}^{2}, \\
& \bar{r} e_{3}^{\prime}=-e_{3}+e_{4}, \\
& \bar{r} e_{4}^{\prime}=\frac{4 \pi \bar{r}^{4} \bar{\rho}^{\prime}}{\bar{m}} e_{2}+2 e_{3}-2 e_{4},
\end{aligned}
$$

in which a prime now indicates differentiation with respect to $\bar{r}$.

An examination of Eqs. (7.24 near $\bar{r}=0$ indicates that the functions $e_{n}$ all tend to a nonvanishing constant at $\bar{r}=0$, and that they admit an expansion in powers of $\bar{r}^{2}$. Furthermore, the local analysis reveals that $e_{1}(0)$ and $e_{3}(0)$ are freely specifiable constants that determine all other coefficients in the power expansions. On the other hand, an examination of the equations near $\bar{r}=1$ shows that except for $e_{1}$, all functions tend to a nonvanishing constant at 


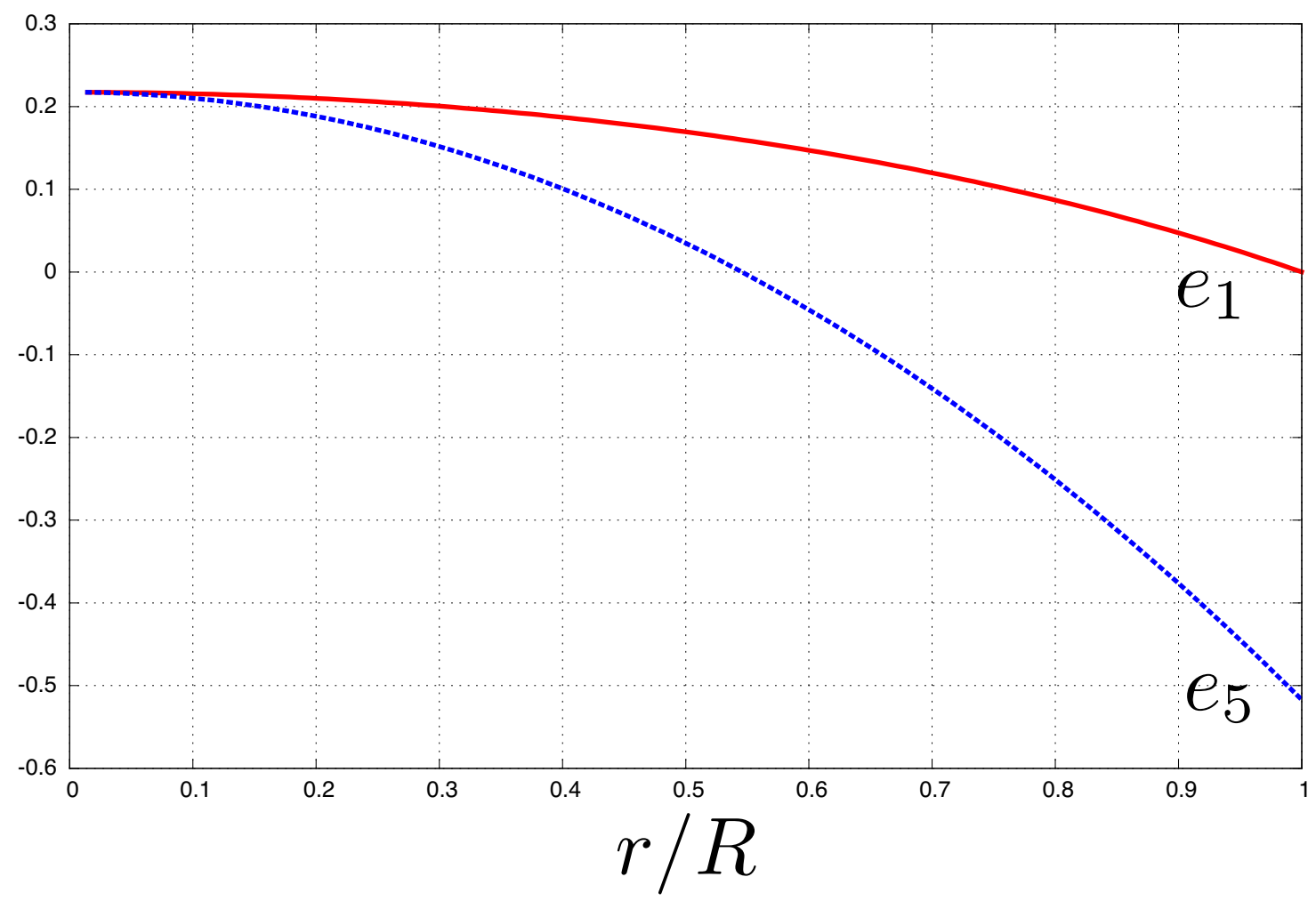

FIG. 1. Numerical solution for the functions $e_{1}$ and $e_{5}$ that substitute for the variables $\mathbf{z}_{r}^{\mathrm{d}}$ and $\mathrm{z}^{\mathrm{d}}$, respectively, plotted as functions of $\bar{r}=r / R$. The numerical error is well within the thickness of the curves.

$\bar{r}=1$; for $e_{1}$ we must impose $e_{1}(1)=0$ to account for the singular factor $\bar{\rho}^{-1}$ in Eq. 7.24a. All functions admit an expansion in powers of $1-\bar{r}$. We also find that $e_{1}^{\prime}(1), e_{3}(1)$, and $e_{4}(1)$ are freely specifiable and determine all other coefficients in the power expansion.

A boundary condition at $\bar{r}=1$ is required to make the problem well posed. As we discuss in Appendix B, the external solution for $\mathrm{U}^{\mathrm{d}}$ must be linear in $\bar{r}$, so that $e_{3}^{\text {ext }}=$ constant. Equation $7.24 \mathrm{c}$ further implies that $e_{4}^{\text {ext }}=e_{3}^{\text {ext }}$, and the required surface condition is therefore that $e_{4}(1)=e_{3}(1)$. With this we have a total of four boundary values that cannot be determined solely from a local analysis near $\bar{r}=0$ and $\bar{r}=1$; these are $e_{1}(0), e_{3}(0), e_{1}^{\prime}(1)$, and $e_{3}(1)$. A global integration is required to determine all four constants, and a practical approach is to shoot toward a middle point: We first integrate the equations from $\bar{r}=0$ up to a middle point $\bar{r}=\bar{r}_{1}$, then integrate them again from $\bar{r}=1$ down to $\bar{r}_{1}$, and search for the boundary values that force the two sets of solutions to agree with each other at $\bar{r}=\bar{r}_{1}$. A concrete implementation of this method is described in Sec. 17.2 of Numerical Recipes [40].

For the numerical work we adopt the polytropic model described by Eqs. (7.1) and (7.2). For this specific case we have

$$
\frac{\bar{r} \bar{\rho}^{\prime}+2 \bar{\rho}}{\bar{\rho}}=\frac{\sin (\pi \bar{r})+(\pi \bar{r}) \cos (\pi \bar{r})}{\sin (\pi \bar{r})}, \quad \frac{4 \pi \bar{r}^{4} \bar{\rho}^{\prime}}{\bar{m}}=-(\pi \bar{r})^{2}
$$

The numerical solutions for $e_{1}, e_{2}, e_{3}$, and $e_{5}$ are displayed in Figs. 1 and 2 the solution for $e_{4}$ is not shown, because it can be obtained directly from $e_{3}$ by exploiting Eq. (7.24c).

According to Eqs. $6.13 \mathrm{e}$ and 7.18 , we have that

$$
w_{r}^{\mathrm{d}}=\mathrm{z}_{r}^{\mathrm{d}} \int_{-\infty}^{t} \mathcal{B}_{\mathrm{m}}^{\mathrm{q}}\left(t^{\prime}\right) d t^{\prime}, \quad w^{\mathrm{d}}=\mathrm{z}^{\mathrm{d}} \int_{-\infty}^{t} \mathcal{B}_{\mathrm{m}}^{\mathrm{q}}\left(t^{\prime}\right) d t^{\prime},
$$

in which we neglected terms proportional to $\partial_{t} \mathcal{B}_{\mathrm{m}}^{\mathrm{q}}$. Substituting this within Eqs. 6.11d and $6.12 \mathrm{~b}$ and recalling 


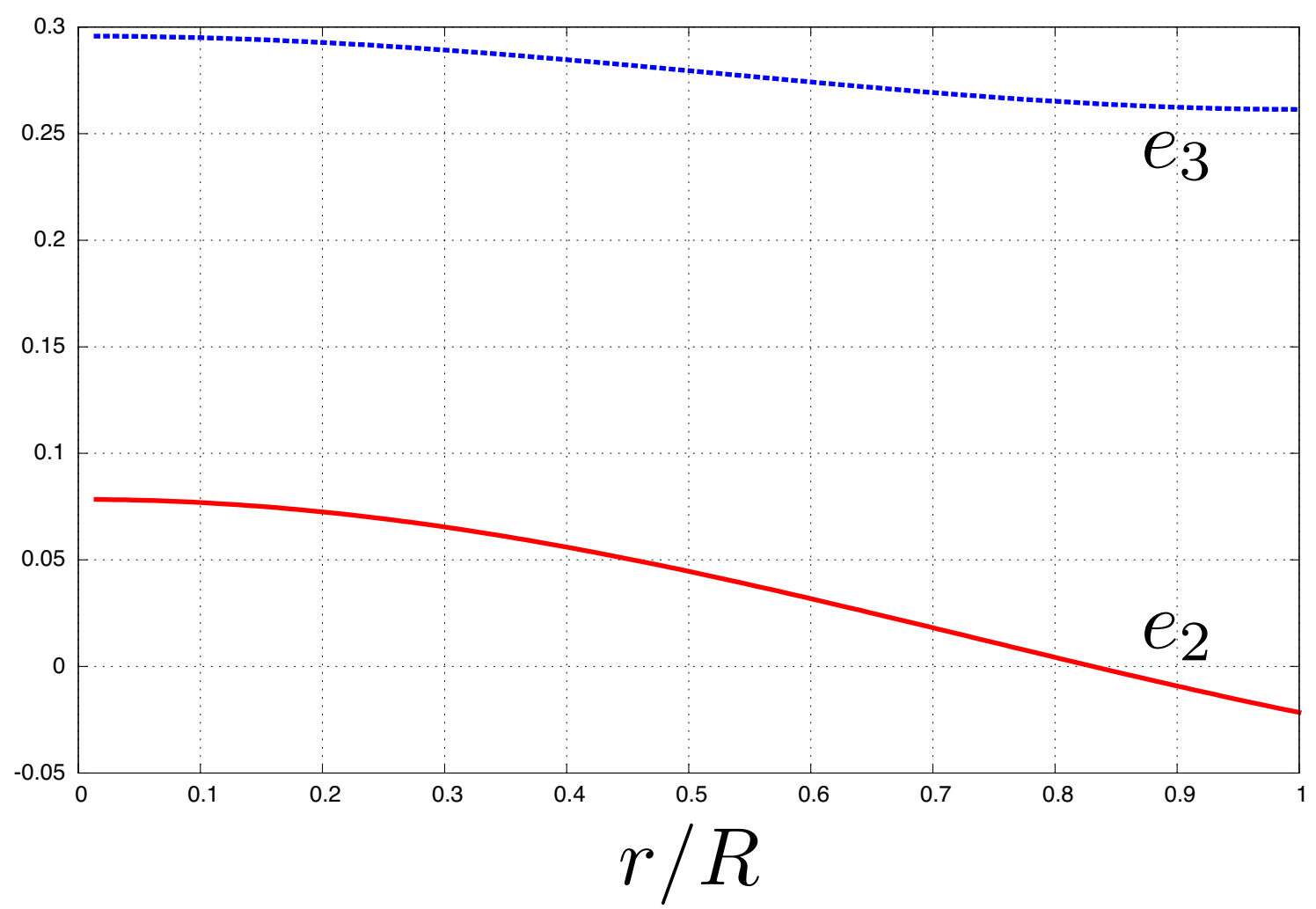

FIG. 2. Numerical solution for the functions $e_{2}$ and $e_{3}$ that substitute for the variables $\mathrm{p}^{\mathrm{d}}$ and $\mathrm{U}^{\mathrm{d}}$, respectively, plotted as functions of $\bar{r}=r / R$. The numerical error is well within the thickness of the curves.

Eqs. 6.5 and 6.8a), we find that the dipole piece of the velocity perturbation is given by

$$
w_{r}^{\ell=1}=\mathrm{z}_{r}^{\mathrm{d}} \int_{-\infty}^{t} \mathcal{K}^{\mathrm{d}}\left(t^{\prime}\right) d t^{\prime}, \quad w_{A}^{\ell=1}=\mathrm{z}^{\mathrm{d}} \int_{-\infty}^{t} \mathcal{K}_{A}^{\mathrm{d}}\left(t^{\prime}\right) d t^{\prime}
$$

If we next express this in terms of the radial functions $e_{1}$ and $e_{5}$ by invoking Eqs. 7.22 and $(7.23)$, and convert to Cartesian coordinates, we get

$$
w_{a}^{\ell=1}=\frac{R^{2}}{c^{2}}\left[e_{1} n_{a} n^{b}+e_{5}\left(\delta_{a}^{b}-n_{a} n^{b}\right)\right] \int_{-\infty}^{t} \mathcal{K}_{b}\left(t^{\prime}\right) d t^{\prime},
$$

where $\mathcal{K}_{a}$ is defined by Eq. 6.2 . For the specific tidal environment provided by a companion body of mass $M^{\prime}$ moving on a circular orbit of radius $r^{\prime}$ in the body's equatorial plane, the quadrupole velocity field is

$$
\begin{aligned}
& w_{x}^{\ell=1}=\frac{3 G M^{\prime} v^{\prime} R^{2}}{2 c^{2} r^{\prime 3}} \frac{\Omega}{\omega^{\prime}}\left\{\sin ^{2} \theta[\sin \Phi+\sin (\Phi-2 \phi)] e_{1}+\left[\left(\cos ^{2} \theta+1\right) \sin \Phi-\sin ^{2} \theta \sin (\Phi-2 \phi)\right] e_{5}\right\}, \\
& w_{y}^{\ell=1}=-\frac{3 G M^{\prime} v^{\prime} R^{2}}{2 c^{2} r^{\prime 3}} \frac{\Omega}{\omega^{\prime}}\left\{\sin ^{2} \theta[\cos \Phi-\cos (\Phi-2 \phi)] e_{1}+\left[\left(\cos ^{2} \theta+1\right) \cos \Phi+\sin ^{2} \theta \sin (\Phi-2 \phi)\right] e_{5}\right\}, \\
& w_{z}^{\ell=1}=\frac{3 G M^{\prime} v^{\prime} R^{2}}{c^{2} r^{\prime}} \frac{\Omega}{\omega^{\prime}} \sin \theta \cos \theta \sin (\Phi-\phi)\left(e_{1}-e_{5}\right),
\end{aligned}
$$

where $v^{\prime}=r^{\prime} \omega^{\prime}$ is the orbital velocity, $\omega^{\prime}$ the orbital angular velocity of Eq. 2.8, $\Phi:=\omega^{\prime} t$ the orbital phase, and $(\theta, \phi)$ the polar angles associated with the coordinates $x^{a}$.

A calculation similar to the one leading to Eq. (7.28) reveals that the dipole piece of the pressure and potential perturbations are given by

$$
\delta p^{\ell=1} / \rho=\frac{R^{2}}{c^{2}} e_{2} \mathcal{K}_{a} x^{a}, \quad \delta U^{\ell=1}=\frac{R^{2}}{c^{2}} e_{3} \mathcal{K}_{a} x^{a} .
$$


In Appendix C we construct the dipole piece of the acceleration field, and verify that its mass-weighted average gives a vanishing acceleration for the body's center-of-mass.

\section{ZERO-FREQUENCY MODES}

In this section we examine the zero-frequency modes $f_{I}^{a}$ introduced in Sec. V] and show that they are directly responsible for the velocity fields displayed in Eqs. (7.6) and (7.28). The completion of the mode analysis initiated in Sec. V provides a complete justification of the assumptions made in Sec. VII concerning the form of solution to the perturbation equations in the dipole and octupole sectors. As in the rest of the paper we assume that the fluid is barotropic, with the perturbed fluid possessing the same equation of state as the unperturbed fluid. In this case it is known (see Ref. 41 for a clear presentation) that the zero-frequency modes separate into even-parity $g$-modes and odd-parity $r$-modes. (The $g$-modes do not exist when the perturbed fluid possesses a distinct equation of state.)

\section{A. Mode equation}

Returning to the notation introduced in Sec. V the zero-frequency modes satisfy $\mathscr{L}_{a}{ }^{b} f_{b} \equiv-P_{a}=0$, which takes the explicit form displayed in Eq. (3.7). Writing the equation in spherical coordinates $(r, \theta, \phi)$, we see that the angular components reduce to $\delta p-\rho \delta U=0$, and that the radial component, simplified with the structure equations $(3.2)$, merely reproduces $\delta p=(d p / d \rho) \delta \rho$. Inserting these relations into Eq 3.12 produces $\nabla^{2} \delta U-\left(4 \pi r^{2} \rho^{\prime} / m\right) \delta U=0$, and it is not difficult to show that the general solution to this equation cannot be smoothly matched to an external solution that is required to decay with increasing $r$. One way to establish this is to perform a decomposition in spherical harmonics, observe that each $\delta U_{\ell m}(r)$ satisfies a homogeneous equation, that the solution regular at $r=0$ comes with a single integration constant (an overall multiplicative factor), and that this single constant is insufficient to match both $\delta U_{\ell m}(r)$ and its first derivative to the external solution $\delta U_{\ell m} \propto r^{-\ell+1}$ at $r=R$.

The conclusion is that the zero-frequency modes describe a perturbation with $\delta \rho=\delta p=\delta U=0$. Equation (4.5) then implies that the mode functions are constrained by

$$
\partial_{a}\left(\rho f^{a}\right)=0 .
$$

A displacement vector $\zeta_{a}=f_{a}$ would describe an entirely trivial perturbation with vanishing $\delta \rho, \delta p, \delta U$, and $\delta v_{a}$. But the displacement vector $\zeta_{a}=f_{a} t$ also satisfies $\partial_{t t} \zeta_{a}-P_{a}=0$, and it does give rise to a nontrivial velocity field $\delta v_{a}=\partial_{t} \zeta_{a}=f_{a}$. A zero-frequency perturbation is therefore a velocity field constrained by Eq. 8.1. .

\section{B. Basis of zero-frequency modes}

We transform Eq. 8.1) to spherical coordinates $\left(r, \theta^{A}\right)$ and consider solutions of the factorized form

$$
f_{r}=f_{r}^{\ell m} Y^{\ell m}, \quad f_{A}=f^{\ell m} Y_{A}^{\ell m}
$$

for the even-parity $g$-modes, and

$$
f_{r}=0, \quad f_{A}=\hat{f}^{\ell m} X_{A}^{\ell m}
$$

for the odd-parity $r$-modes, where $Y^{\ell m}, Y_{A}^{\ell m}$, and $X_{A}^{\ell m}$ are the spherical harmonics introduced in Sec. VI the functions $f_{r}^{\ell m}, f^{\ell m}$, and $\hat{f}^{\ell m}$ depend on $r$ only. Making the substitutions in Eq. 8.1 reveals that the $g$-mode functions are constrained by

$$
\ell(\ell+1) \rho f^{\ell m}=\frac{d}{d r}\left(r^{2} \rho f_{r}^{\ell m}\right),
$$

so that $f^{\ell m}$ is determined once $f_{r}^{\ell m}$ is specified. The exercise further reveals that the $r$-mode function is completely unconstrained. The zero-frequency modes are therefore characterized by two freely specifiable functions, $f_{r}^{\ell m}$ and $\hat{f}^{\ell m}$. We have two infinitely degenerate sets of modes.

Two $g$-modes, $\boldsymbol{a}$ and $\boldsymbol{b}$, which share the same values of $\ell$ and $\mathrm{m}$, have a scalar product defined by

$$
\langle\boldsymbol{a}, \boldsymbol{b}\rangle:=\int \rho \boldsymbol{a} \cdot \boldsymbol{b} d^{3} x=N^{\ell \mathrm{m}}\left[\int_{0}^{R} \rho a_{r}^{\ell \mathrm{m}} b_{r}^{\ell \mathrm{m}} r^{2} d r+\ell(\ell+1) \int_{0}^{R} \rho a^{\ell \mathrm{m}} b^{\ell \mathrm{m}} d r\right],
$$


where $N^{\ell \mathrm{m}}:=\int\left(Y^{\ell \mathrm{m}}\right)^{2} \sin \theta d \theta d \phi$; modes with different values of either $\ell$ or $\mathrm{m}$ are orthogonal. Similarly, $r$-modes $\boldsymbol{p}$ and $\boldsymbol{q}$ have the scalar product

$$
\langle\boldsymbol{p}, \boldsymbol{q}\rangle=\ell(\ell+1) N^{\ell \mathrm{m}} \int \rho \hat{p}^{\ell \mathrm{m}} \hat{q}^{\ell \mathrm{m}} d r
$$

when they share the same values of $\ell$ and $\mathrm{m}$. All $g$-modes are orthogonal to all $r$-modes.

We wish to construct a basis of orthogonal modes, labelled by $k=0,1,2, \cdots$ in addition to the spherical-harmonic labels $\ell \mathrm{m}$; the complete mode label is therefore $I:=\ell \mathrm{m} k$. The procedure is simple, and we describe it in detail in the case of $g$-modes. We begin with a set of seed modes $\boldsymbol{a}^{k}$ characterized by a freely-specified $a_{r}^{\ell m k}$ and an $a^{\ell \mathrm{m} k}$ determined by Eq. (8.4). These modes are not mutually orthogonal, but they can be turned into a set of orthogonal modes by implementing a Gram-Schmidt procedure. We first set $\boldsymbol{f}^{0}=\boldsymbol{a}^{0}$, and then set

$$
f_{r}^{\ell m k}=a_{r}^{\ell m k}-\sum_{n=0}^{k-1} \frac{\left\langle\boldsymbol{f}^{n}, \boldsymbol{a}^{k}\right\rangle}{\left\langle\boldsymbol{f}^{n}, \boldsymbol{f}^{n}\right\rangle} a_{r}^{\ell m n}
$$

for each successive $k$, with $f^{\ell \mathrm{m} k}$ determined at each stage by Eq. 8.4.

The overlap integrals of Eq. 5.5 can now be evaluated. Again we describe the procedure in detail in the case of $g$-modes. The components of the external force $\boldsymbol{B}$ are expanded in spherical harmonics according to

$$
B_{r}=\sum_{\ell m} B_{r}^{\ell m} Y^{\ell m}, \quad B_{A}=\sum_{\ell m} B^{\ell m} Y_{A}^{\ell m}+\sum_{\ell m} \hat{B}^{\ell m} X_{A}^{\ell m}
$$

and Eq. 5.5 turns into the explicit form

$$
B^{\ell \mathrm{m} k}=\frac{N^{\ell \mathrm{m}}}{\left\langle\boldsymbol{f}^{k}, \boldsymbol{f}^{k}\right\rangle}\left[\int_{0}^{R} \rho B_{r}^{\ell \mathrm{m}} f^{\ell \mathrm{m} k} r^{2} d r+\ell(\ell+1) \int_{0}^{R} \rho B^{\ell \mathrm{m}} f^{\ell \mathrm{m} k} d r\right]
$$

for the mode amplitudes $B^{I}$. This can be simplified by inserting Eq. 8.4 within the second integral and integrating by parts; we arrive at

$$
B^{\ell \mathrm{m} k}=\frac{N^{\ell \mathrm{m}}}{\left\langle\boldsymbol{f}^{k}, \boldsymbol{f}^{k}\right\rangle} \int_{0}^{R} \rho\left(B_{r}^{\ell \mathrm{m}}-\frac{d B^{\ell \mathrm{m}}}{d r}\right) f_{r}^{\ell \mathrm{m} k} r^{2} d r .
$$

With this, the components of $\boldsymbol{B}^{\text {zf }}$ defined below Eq. [5.9) are given by

$$
B_{r}^{\mathrm{zf}}=\sum_{\ell \mathrm{m} k} B^{\ell \mathrm{m} k} f_{r}^{\ell \mathrm{m} k} Y^{\ell \mathrm{m}}, \quad B_{A}^{\mathrm{zf}}=\sum_{\ell \mathrm{m} k} B^{\ell \mathrm{m} k} f^{\ell \mathrm{m} k} Y_{A}^{\ell \mathrm{m}},
$$

and these are then ready to be inserted within Eq. (5.9) to obtain the growing piece of the velocity perturbation.

\section{Quadrupole sector}

According to Eq. (6.9), the quadrupole piece of the driving force $\boldsymbol{B}$ has the nonvanishing components

$$
B_{A}^{\ell=2}=\frac{1}{9} r^{3} \hat{\mathcal{B}}_{A}^{\mathrm{q}},
$$

and these admit a decomposition in odd-parity harmonics $X_{A}^{2, \mathrm{~m}}$ with coefficients $\hat{B}^{2, \mathrm{~m}}$. Because the $r$-modes are unconstrained, we have the freedom to assign $\hat{f}^{2, \mathrm{~m}, 0}=\hat{B}^{2, \mathrm{~m}}$. And because all other members of the basis of modes are orthogonal to the zeroth member, we immediately find that

$$
\boldsymbol{B}_{\mathrm{zf}}^{\ell=2}=\boldsymbol{B}^{\ell=2} .
$$

The growing solution of Eq. (5.8) can then be seen to give rise to the velocity field of Eq. (7.5). In this specific case the mode analysis is entirely trivial and merely reproduces our previous results. 


\section{Octupole sector}

Returning to Eq. (6.9), we see that the octupole piece of the driving force has the components

$$
B_{r}^{\ell=3}=-\frac{1}{6} r^{2} \mathcal{K}^{\circ}, \quad B_{A}^{\ell=3}=-\frac{1}{6} r^{3} \mathcal{K}_{A}^{\circ},
$$

which admit a decomposition in even-parity harmonics $Y^{3, \mathrm{~m}}$ and $Y_{A}^{3, \mathrm{~m}}$ with coefficients $B_{r}^{3, \mathrm{~m}}=-\frac{1}{6} r^{2} \mathcal{K}_{\mathrm{m}}^{\circ}$ and $B^{3, \mathrm{~m}}=$ $-\frac{1}{18} r^{3} \mathcal{K}_{\mathrm{m}}^{\circ}$, respectively. These are related by

$$
B_{r}^{3, \mathrm{~m}}-\frac{d B^{3, \mathrm{~m}}}{d r}=0
$$

and Eq. 8.10 implies that all mode amplitudes $B^{3, \mathrm{~m}, k}$ necessarily vanish. In this case we have that

$$
\boldsymbol{B}_{\mathrm{zf}}^{\ell=3}=0,
$$

and this justifies the assumption made at the beginning of Sec. VIIB, that the octupole piece of the velocity field does not possess a growing term.

\section{E. Dipole sector}

Returning once more to Eq. 6.9 , we see that the dipole piece of the driving force has the components

$$
B_{r}^{\ell=1}=\frac{1}{10} r^{2} \mathcal{K}^{\mathrm{d}}, \quad B_{A}^{\ell=1}=\frac{1}{5} r^{3} \mathcal{K}_{A}^{\mathrm{d}},
$$

which admit a decomposition in even-parity harmonics with coefficients $B_{r}^{1, \mathrm{~m}}=\frac{1}{10} r^{2} \mathcal{K}_{\mathrm{m}}^{\mathrm{d}}$ and $B^{1, \mathrm{~m}}=\frac{1}{5} r^{3} \mathcal{K}_{\mathrm{m}}^{\mathrm{d}}$, respectively. We choose the mode functions to be independent of $\mathrm{m}$, denote them $f_{r}^{k}$ and $f^{k}$ to simplify the notation (with the label $\ell=1$ omitted), and find that in this case, the mode amplitudes are given by

$$
B^{1, \mathrm{~m}, k}=-\frac{1}{2} \mathcal{K}_{\mathrm{m}}^{\mathrm{d}} \Gamma_{k},
$$

where

$$
\Gamma_{k}:=\frac{N^{1, \mathrm{~m}}}{\left\langle\boldsymbol{f}^{k}, \boldsymbol{f}^{k}\right\rangle} \int_{0}^{R} \rho r^{4} f_{r}^{k} d r
$$

these quantities are independent of $\mathrm{m}$ by virtue of the definition of the scalar product in Eq. 8.5. With this we have that

$$
B_{r}^{\mathrm{zf}}=-\frac{1}{2} \mathcal{K}^{\mathrm{d}} \sum_{k} \Gamma_{k} f_{r}^{k}, \quad B_{A}^{\mathrm{zf}}=-\frac{1}{2} \mathcal{K}_{A}^{\mathrm{d}} \sum_{k} \Gamma_{k} f^{k},
$$

and substitution into Eq. 5.9 produces the velocity field of Eq. 7.27 , with $\mathbf{z}_{r}^{\mathrm{d}}=\left(2 / c^{2}\right) \sum_{k} \Gamma_{k} f_{r}^{k}$ and $\mathbf{z}^{\mathrm{d}}=$ $\left(2 / c^{2}\right) \sum_{k} \Gamma_{k} f^{k}$. With the definitions of Eqs. 7.22 and 7.23 , this is

$$
e_{1}=\frac{2}{R^{2}} \sum_{k} \Gamma_{k} f_{r}^{k}, \quad r e_{5}=\frac{2}{R^{2}} \sum_{k} \Gamma_{k} f^{k} .
$$

At this stage we have justified the assumption made at the beginning of Sec. VIIC that the dipole piece of the velocity field possesses a growing term. In addition, the mode equation (8.4) implies that $e_{1}$ and $e_{5}$ are related by $2 r \rho e_{5}=\left(r^{2} \rho e_{1}\right)^{\prime}$, with a prime indicating differentiation with respect to $r$. This relation can also be derived on the basis of Eq. 7.24a), and we see that the mode analysis is entirely compatible with the developments of Sec. VIIC.

We have yet to verify that the $e_{1}$ constructed here is precisely equal to the $e_{1}$ obtained in Sec. VIIC For this we must introduce an actual set of modes $f^{k}$, calculate the overlap integrals $\Gamma_{k}$, and carry out the sum over modes. To accomplish this we adopt

$$
a_{r}^{k}=\cos \left[\frac{1}{2}(2 k+1) \pi r / R\right], \quad k=0,1,2, \cdots
$$




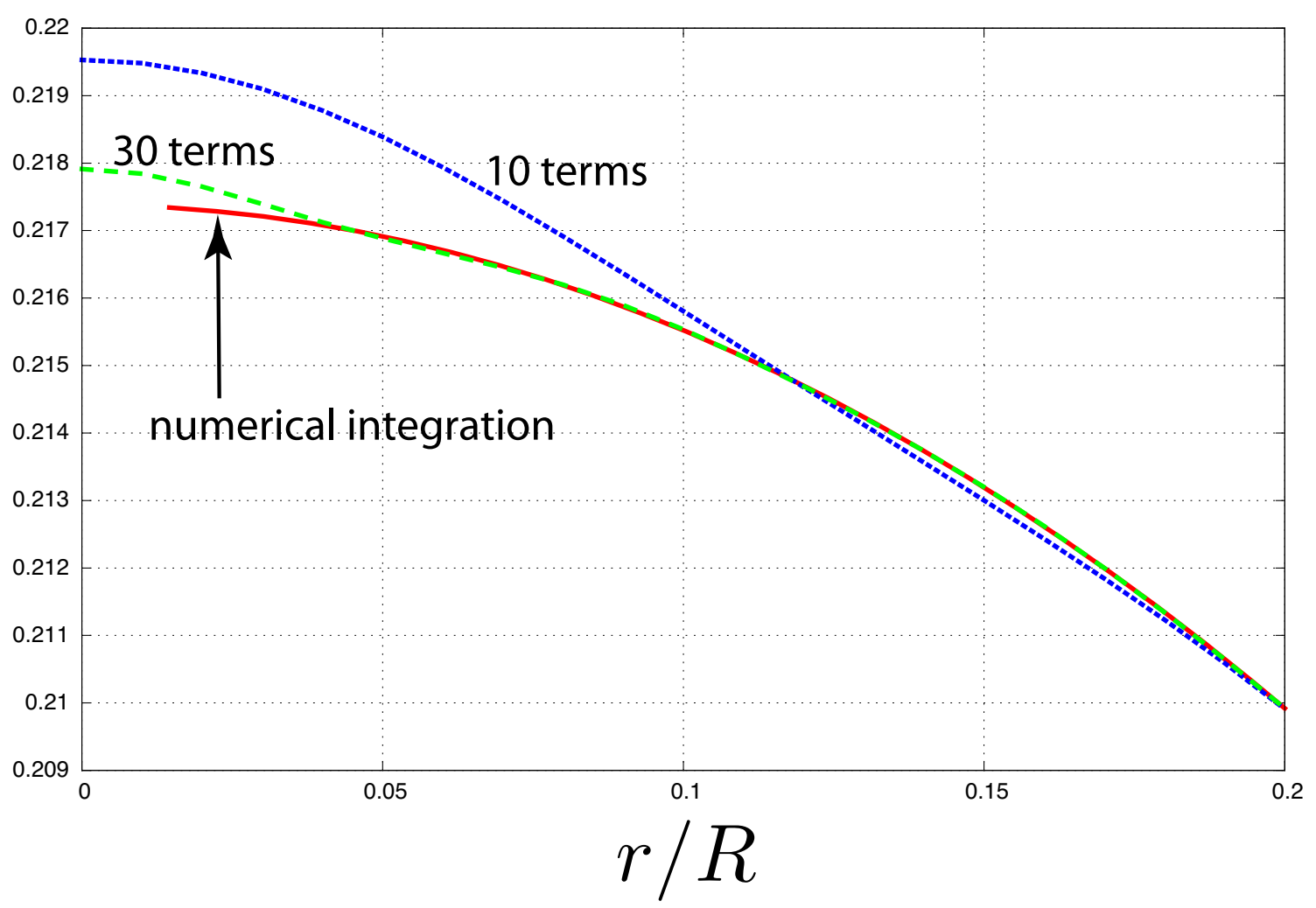

FIG. 3. Mode-sum representation of $e_{1}$ compared with the result displayed in Fig. 1. The curve obtained by numerical intergation in Sec. VIIC is shown in solid red. A construction of $e_{1}$ involving 10 terms in the mode sum is shown in short dashed blue. A construction involving 30 terms is shown in long dashed green. The plot focuses on the interval $0<r / R<0.2$; the curves are indistinguishable beyond this interval.

as a convenient set of seed modes, with the corresponding $a^{k}$ determined by Eq. (8.4). These mode functions are chosen so that $a_{r}^{k}$ vanishes at $r=R$, as required by the mode equation in view of the fact that $\rho$ vanishes at the surface, that it tends to a nonvanishing constant at $r=0$, as required of a dipolar vector field, and that its derivative with respect to $r$ vanishes at $r=0$, as expected of $e_{1}(r)$. We feed the seed modes into the Gram-Schmidt machine, using the density function of Eq. 77.1 to evaluate the integrals, and obtain a set of orthogonal modes $\boldsymbol{f}^{k}$. These, finally, are involved in the computation of $\Gamma_{k}$ and the mode sum of Eq. (8.21).

At the end of this computation we find that indeed, the $e_{1}$ of Eq. (8.21) agrees with the $e_{1}$ displayed in Fig. 1. the comparison is shown in Fig. 3. The mode sum converges rapidly when $r / R \gtrsim 0.2$; in this range a handful of terms suffice to produce a curve that is visually identical to the one shown in Fig. 11. The sum converges much more slowly when $r$ is small; we find that at least thirty terms are required to adequately reproduce the curve near the smallest values of $r$ displayed in Fig. 1 .

\section{F. Conclusion}

We have shown that the zero-frequency modes are directly responsible for the velocity fields displayed in Eqs. (7.6) and (7.28). These modes, therefore, play a crucial role in the gravitomagnetic tidal response of a rotating body. Zero-frequency modes, however, are typically not involved in the response of a Newtonian fluid driven by an external force, and they are rarely given consideration. The reason is that in the typical case, the external force $B_{a}$ is the gradient of a scalar potential, $B_{a}=\partial_{a} \psi$, and this guarantees that the corresponding mode amplitudes $B_{I}$ vanish:

$$
N_{I} B_{I}=\int_{V} \rho \boldsymbol{f}_{I} \cdot \nabla \psi d^{3} x=\oint_{S} \rho \psi \boldsymbol{f}_{I} \cdot d \boldsymbol{a}-\int_{V} \psi \boldsymbol{\nabla} \cdot\left(\rho \boldsymbol{f}_{I}\right) d^{3} x=0
$$

The integration domain $V$, bounded by the surface $S$, is chosen to extend slightly beyond the body, which ensures that $\rho$ always vanishes on $S$; the second volume integral vanishes by virtue of Eq. (8.1). The zero-frequency modes 
are involved in the gravitomagnetic tidal response of a rotating body because in this case, the driving force $B_{a}$ is not a gradient vector field.

\section{ACKNOWLEDGMENTS}

We thank John Friedman, Phil Landry, Raissa Mendes, and Jean-Philippe Nicolas for useful conversations, and Raissa Mendes again for a helpful hand at integrating Eqs. (7.24). One of us (EP) is grateful for the warm hospitality of the Laboratoire de Mathématiques at the Université de Bretagne Occidentale, where part of this work was carried out. The work was supported by the Natural Sciences and Engineering Research Council of Canada.

\section{Appendix A: Octupole rotational-tidal Love number}

The octupole, rotational-tidal Love number $\mathfrak{K}^{\circ}$ was introduced in Secs. III and IV of Ref. [39]. As explained there, the Love number provides a (partial) description of the body's gravitational response to the coupled rotational and gravitomagnetic tidal perturbations. For our purposes here, the Love number is defined by their Eq. (4.4), in which the external metric of a slowly rotating, tidally deformed body is presented in Regge-Wheeler gauge. The relevant term in $g_{t t}$ is

$$
\delta g_{t t}^{\ell=3}=-\frac{8 G}{c^{6}}\left(\frac{2 G M}{c^{2} r}\right)^{4} \mathfrak{K}^{\circ} S_{\langle a} \mathcal{B}_{b c\rangle} n^{a} n^{b} n^{c}
$$

in which we have replaced $\chi_{a}$ by $S_{a} / M^{2}$, where $S_{a}$ is the body's spin angular momentum, restored factors of $G$ and $c$, and neglected all higher post-Newtonian corrections. This result can be expressed as

$$
\delta U_{\mathrm{eff}}^{\ell=3}=-\frac{4 G}{c^{4}}\left(\frac{2 G M}{c^{2} r}\right)^{4} \mathfrak{K}^{\circ} S_{\langle a} \mathcal{B}_{b c\rangle} n^{a} n^{b} n^{c}
$$

if we introduce an effective gravitational potential via $g_{t t}=-1+2 U_{\mathrm{eff}} / c^{2}$. In our post-Newtonian treatment, the body's spin $S^{a}$ is related to its angular velocity $\Omega^{a}$ through the moment of inertia $I$, given by

$$
I=\frac{8 \pi}{3} \int \rho r^{4} d r
$$

The relation is $S^{a}=I \Omega^{a}$, and making the substitution in Eq. A2 yields

$$
\delta U_{\mathrm{eff}}^{\ell=3}=-\frac{2}{c^{2}}\left(\frac{2 G M}{c^{2}}\right)^{5} \frac{I}{M R^{2}} \mathfrak{K}^{\circ} \frac{R^{2}}{r^{4}} \mathcal{K}_{a b c} n^{a} n^{b} n^{c}
$$

with $\mathcal{K}_{a b c}$ defined by Eq. 6.2 .

This external expression for the octupole gravitational perturbation must be matched to the internal expression of Eq. 7.15 at $r=R$, and this provides the value of $\mathfrak{K}^{\circ}$ for the polytropic model considered in this work. In this case the moment of inertia evaluates to $I /\left(M R^{2}\right)=2\left(\pi^{2}-6\right) /\left(3 \pi^{2}\right)$, and we arrive at

$$
\mathfrak{K}^{\circ}=-\mathfrak{k}^{\circ}\left(\frac{c^{2} R}{2 G M}\right)^{5}, \quad \mathfrak{k}^{\circ}=\frac{5}{18} \frac{21-2 \pi^{2}}{\pi^{2}-6} \simeq 9.0505 \times 10^{-2} .
$$

Making the substitution in Eq. A2 gives

$$
\delta U_{\mathrm{eff}}^{\ell=3}=\frac{2 \mathfrak{k}^{\circ}}{c^{2}} \frac{R^{5}}{r^{4}} \hat{S}_{\langle a} \mathcal{B}_{b c\rangle} n^{a} n^{b} n^{c}
$$

where $\hat{S}_{a}:=S_{a} / M$ is the body's spin per unit mass. The expression reveals that the body's response to a coupled rotational and gravitomagnetic tidal perturbation is a post-Newtonian effect that scales with $R^{5}$. 


\section{Appendix B: External dipole}

In this Appendix we justify the boundary condition $e_{4}(1)=e_{3}(1)$ imposed in Sec. VII C to integrate the perturbation equations in the dipole sector. The condition derives from the statement that in the body's exterior, $\mathrm{U}^{\mathrm{d}} \propto r$ : the dipole piece of $\delta U$ grows linearly with the distance to the body's center-of-mass. We note first that the external perturbation satisfies

$$
r^{2} \frac{d^{2} \mathrm{U}^{\mathrm{d}}}{d r^{2}}+2 r \frac{d U^{\mathrm{d}}}{d r}-2 \mathrm{U}^{\mathrm{d}}=0
$$

with the linearly independent solutions $\mathrm{U}^{\mathrm{d}} \propto r$ and $\mathrm{U}^{\mathrm{d}} \propto 1 / r^{2}$. Our boundary condition states that we must keep the growing solution and reject the decaying solution.

The justification of this statement comes from an examination of the external metric of a slowly rotating body subjected to a gravitomagnetic tidal field. This metric is presented to all post-Newtonian order in Ref. 39], and the first post-Newtonian approximation of the relevant component $g_{t t}$ is displayed in Eq. (8.17a) of Ref. [38. We have

$$
\delta g_{t t}^{\ell=1}=\frac{2}{c^{4}} \mathcal{B}_{a b} \hat{S}^{b} x^{a}
$$

where $\hat{S}^{b}:=S^{b} / M$ is the body's spin angular momentum per unit mass. (This expression was derived in Ref. 38 ] for the specific case of a black hole, but at first post-Newtonian order it applies equally well to any material body.) As explained in detail in Ref. 38, this growing term is intimately tied to the fact that the body does not follow a geodesic in the external spacetime of the remote objects responsible for the tidal field, but is in fact accelerated in this spacetime; its acceleration vector - the acceleration of the body's local frame relative to the global, barycentric frame - is given by $-\mathcal{B}_{a b} \hat{S}^{b} / c^{2}$, a form of the well-known Mathisson-Papapetrou spin force, which gives rise to the spin-orbit and spin-spin acceleration of a rotating body (see Sec. 9.5 of Gravity [32]).

Defining an effective gravitational potential as in Appendix A, the preceding discussion implies that this potential possesses a dipole perturbation

$$
\delta U_{\mathrm{eff}}^{\ell=1}=\frac{1}{c^{2}} \mathcal{B}_{a b} \hat{S}^{b} x^{a}
$$

in the body's exterior. As we have seen, this growing term is tied to the failure of the body to move on a geodesic in the external spacetime of the remote objects. On the other hand, the absence of a decaying term is tied to the definition of the body's center-of-mass. In an analogous Newtonian discussion, a multipole expansion of the potential would normally contain a decaying, dipole term of the form $G p_{a} x^{a} / r^{3}$, with $p^{a}=\int \rho x^{a} d^{3} x$ representing the mass dipole moment. But such a term is eliminated with a judicious choice of center-of-mass, which enforces $p_{a}=0$. In the relativistic setting considered here, the choice of center-of-mass is made implicitly by demanding the absence of a decaying term in $\delta U_{\text {eff }}^{\ell=1}$.

Inserting $S^{a}=I \Omega^{a}$ into Eq. (B3) — refer to Appendix A — and incorporating the definition of Eq. (6.2), we find that

$$
\delta U_{\mathrm{eff}}^{\ell=1}=\frac{I}{M c^{2}} \mathcal{K}_{a} x^{a} .
$$

Comparison with the expression of Eq. 7.30 implies that $e_{3}=I /\left(M R^{2}\right)$ in the body's exterior. We have therefore arrived at the appropriate surface condition for the internal $e_{3}$.

For the polytropic model adopted in the main text, the moment of inertia evaluates to $I /\left(M R^{2}\right)=2\left(\pi^{2}-6\right) /\left(3 \pi^{2}\right) \simeq$ 0.26138193210. The numerical search described in Sec. VIIC returned $e_{3}(1) \simeq 0.26138193211$ for the same quantity. The relative numerical error is of the order of $10^{-10}$, in line with the expectations placed on the code.

\section{Appendix C: Acceleration of the center-of-mass}

The dipole velocity field of Eq. (7.28) gives rise to the acceleration field

$$
a_{a}^{\ell=1}:=\partial_{t} w_{a}^{\ell=1}=\frac{R^{2}}{c^{2}}\left[e_{1} n_{a} n^{b}+e_{5}\left(\delta_{a}^{b}-n_{a} n^{b}\right)\right] \mathcal{K}_{b} .
$$

We aim to prove that this yields a vanishing acceleration for the body's center-of-mass,

$$
a_{a}^{\mathrm{CM}}=\frac{1}{M} \int \rho a_{a}^{\ell=1} d^{3} x
$$


We begin by making the substitution and carrying out the angular integrals, using the identity $(4 \pi)^{-1} \int n_{a} n^{b} \sin \theta d \theta d \phi=$ $\frac{1}{3} \delta_{a}^{b}$. This returns

$$
a_{a}^{\mathrm{CM}}=\frac{4 \pi}{3} \frac{R^{2}}{c^{2}} \mathcal{K}_{a} \int_{0}^{1} \bar{\rho}\left(e_{1}+2 e_{5}\right) \bar{r}^{2} d \bar{r},
$$

in which $\bar{\rho}:=R^{3} \rho / M$ and $\bar{r}:=r / R$. The definition of $e_{5}$ in Eq. 7.23) and the differential equation 7.24a imply that the integrand is

$$
\bar{\rho}\left(e_{1}+2 e_{5}\right) \bar{r}^{2}=\left(\bar{r}^{3} \bar{\rho} e_{1}\right)^{\prime}
$$

in which a prime indicates differentiation with respect to $\bar{r}$. Integration is immediate, and the vanishing of $\bar{\rho}$ at $\bar{r}=1$ guarantees that

$$
a_{a}^{\mathrm{CM}}=0
$$

[1] E. E. Flanagan and T. Hinderer, Constraining neutron star tidal Love numbers with gravitational wave detectors, Phys. Rev. D 77, 021502(R) (2008), arXiv:0709.1915.

[2] T. Hinderer, Tidal Love numbers of neutron stars, Astrophys. J. 677, 1216 (2008), erratum: Astrophys. J. 697, 964 (2009), arXiv:0711.2420.

[3] T. Damour and A. Nagar, Relativistic tidal properties of neutron stars, Phys. Rev. D 80, 084035 (2009), arXiv:0906.0096.

[4] T. Binnington and E. Poisson, Relativistic theory of tidal Love numbers, Phys. Rev. D 80, 084018 (2009), arXiv:0906.1366.

[5] P. Landry and E. Poisson, Relativistic theory of surficial Love numbers, Phys. Rev. D 89, 124011 (2014), arXiv:1404.6798.

[6] T. Hinderer, B. D. Lackey, R. N. Lang, and J. S. Read, Tidal deformability of neutron stars with realistic equations of state and their gravitational wave signatures in binary inspiral, Phys. Rev. D 81, 123016 (2010), arXiv:0911.3535.

[7] L. Baiotti, T. Damour, B. Giacomazzo, A. Nagar, and L. Rezzolla, Analytic modeling of tidal effects in the relativistic inspiral of binary neutron stars, Phys. Rev. Lett. 105, 261101 (2010), arXiv:1009.0521.

[8] L. Baiotti, T. Damour, B. Giacomazzo, A. Nagar, and L. Rezzolla, Accurate numerical simulations of inspiralling binary neutron stars and their comparison with effective-one-body analytical models, Phys. Rev. D 84, 024017 (2011), arXiv:1103.3874.

[9] J. Vines, E. E. Flanagan, and T. Hinderer, Post-1-Newtonian tidal effects in the gravitational waveform from binary inspirals, Phys. Rev. D 83, 084051 (2011), arXiv:1101.1673.

[10] F. Pannarale, L. Rezzolla, F. Ohme, and J. S. Read, Will black hole-neutron star binary inspirals tell us about the neutron star equation of state?, Phys. Rev. D 84, 104017 (2011), arXiv:1103.3526.

[11] B. D. Lackey, K. Kyutoku, M. Shibata, P. R. Brady, and J. L. Friedman, Extracting equation of state parameters from black hole-neutron star mergers. I. Nonspinning black holes, Phys. Rev. D 85, 044061 (2012), arXiv:1109.3402.

[12] T. Damour, A. Nagar, and L. Villain, Measurability of the tidal polarizability of neutron stars in late-inspiral gravitationalwave signals, Phys. Rev. D 85, 123007 (2012), arXiv:1203.4352.

[13] D. Bini, T. Damour, and G. Faye, Effective action approach to higher-order relativistic tidal interactions in binary systems and their effective one body description, Phys. Rev. D 85, 124034 (2012), arXiv:1202.3565.

[14] J. S. Read, L. Baiotti, J. D. E. Creighton, J. L. Friedman, B. Giacomazzo, K. Kyutoku, C. Markakis, L. Rezzolla, M. Shibata, and K. Taniguchi, Matter effects on binary neutron star waveforms, Phys. Rev. D 88, 044042 (2013), arXiv:1306.4065.

[15] J. E. Vines and E. E. Flanagan, First-post-Newtonian quadrupole tidal interactions in binary systems, Phys. Rev. D 88, 024046 (2013), arXiv:1009.4919.

[16] A. Maselli, L. Gualtieri, and V. Ferrari, Constraining the equation of state of nuclear matter with gravitational wave observations: Tidal deformability and tidal disruption, Phys. Rev. D 88, 104040 (2013), arXiv:1310.5381.

[17] S. Chakrabarti, T. Delsate, and J. Steinhoff, New perspectives on neutron star and black hole spectroscopy and dynamic tides (2013), arXiv:1304.2228.

[18] S. Chakrabarti, T. Delsate, and J. Steinhoff, Effective action and linear response of compact objects in Newtonian gravity, Phys. Rev. D 88, 084038 (2013), arXiv:1306.5820.

[19] B. D. Lackey, K. Kyutoku, M. Shibata, P. R. Brady, and J. L. Friedman, Extracting equation of state parameters from black hole-neutron star mergers: Aligned-spin black holes and a preliminary waveform model, Phys. Rev. D 89, 043009 (2014), arXiv:1303.6298.

[20] S. R. Dolan, P. Nolan, A. C. Ottewill, N. Warburton, and B. Wardell, Tidal invariants for compact binaries on quasicircular orbits, Phys. Rev. D 91, 023009 (2015), arXiv:1406.4890.

[21] D. Bini and T. Damour, Gravitational self-force corrections to two-body tidal interactions and the effective one-body formalism, Phys. Rev. D 90, 124037 (2014), arXiv:1409.6933.

[22] M. Favata, Systematic parameter errors in inspiraling neutron star binaries, Phys. Rev. Lett. 112, 101101 (2014), arXiv:1310.8288. 
[23] K. Yagi and N. Yunes, Love number can be hard to measure, Phys. Rev. D 89, 021303 (2014), arXiv:1310.8358.

[24] T. Delsate, I-Love relations for irrotational stars, Phys. Rev. D 92, 124001 (2015), arXiv:1504.07335.

[25] P. Pani, L. Gualtieri, A. Maselli, and V. Ferrari, Tidal deformations of a spinning compact object, Phys. Rev. D 92, 024010 (2015), arXiv:1503.07365.

[26] P. Pani, L. Gualtieri, and V. Ferrari, Tidal Love numbers of a slowly spinning neutron star, Phys. Rev. D 92, 124003 (2015), arXiv:1509.02171.

[27] V. Ferrari, L. Gualtieri, and A. Maselli, Tidal interaction in compact binaries: A post-Newtonian affine framework, Phys. Rev. D 85, 044045 (2012), arXiv:1111.6607.

[28] A. Maselli, L. Gualtieri, F. Pannarale, and V. Ferrari, On the validity of the adiabatic approximation in compact binary inspirals, Phys. Rev. D 86, 044032 (2012), arXiv:1205.7006.

[29] A. Maselli, V. Cardoso, V. Ferrari, L. Gualtieri, and P. Pani, Equation-of-state-independent relations in neutron stars, Phys. Rev. D 88, 023007 (2013), arXiv:1304.2052.

[30] J. Steinhoff, T. Hinderer, A. Buonanno, and A. Taracchini, Dynamical tides in general relativity: Effective action and effective-one-body Hamiltonian, Phys. Rev. D 94, 104028 (2016), arXiv:1608.01907.

[31] P. Landry and E. Poisson, Dynamical response to a stationary tidal field, Phys. Rev. D 92, 124041 (2015), arXiv:1510.09170.

[32] E. Poisson and C. M. Will, Gravity: Newtonian, Post-Newtonian, Relativistic (Cambridge University Press, Cambridge, England, 2014).

[33] X.-H. Zhang, Multipole expansions of the general-relativistic gravitational field of the external universe, Phys. Rev. D 34, 991 (1986).

[34] S. L. Shapiro, Gravitomagnetic Induction during the Coalescence of Compact Binaries, Phys. Rev. Lett. 77, 4487 (1996).

[35] M. Favata, Are neutron stars crushed? Gravitomagnetic tidal fields as a mechanism for binary-induced collapse, Phys. Rev. D 73, 104005 (2006), arXiv:astro-ph/0510668.

[36] S. Chandrasekhar, A general variational principle governing the radial and the non-radial oscillations of gaseous masses, Astrophys. J. 139, 664 (1964).

[37] H. R. Beyer and B. G. Schmidt, Newtonian stellar oscillations, Astron. Astrophys. 296, 722 (1995).

[38] E. Poisson, Tidal deformation of a slowly rotating black hole, Phys. Rev. D 91, 044004 (2015), arXiv:1411.4711.

[39] P. Landry and E. Poisson, Tidal deformation of a slowly rotating material body: External metric, Phys. Rev. D 91, 104018 (2015), arXiv:1503.07366.

[40] W. H. Press, S. A. Teukolsky, W. T. Vetterling, and B. P. Flannery, Numerical Recipes in C++: The Art of Scientific Computing, Second Edition (Cambridge University Press, Cambridge, England, 2002).

[41] K. H. Lockitch, N. Andersson, and J. L. Friedman, Rotational modes of relativistic stars: Analytic results, Phys. Rev. D 63, 024019 (2000), arXiv:gr-qc/0008019. 